\title{
Proton-transfer-induced 3D/2D hybrid perovskites suppress ion migration and reduce luminance overshoot
}

\author{
Hobeom Kim (1) 1,17, Joo Sung Kim (1) 1,17, Jung-Min Heo (1) 1,17, Mingyuan Pei², In-Hyeok Park (1) ${ }^{3}$, Zhun Liu ${ }^{4}$, \\ Hyung Joong Yun ${ }^{5}$, Min-Ho Park1, Su-Hun Jeong1, Young-Hoon Kim¹, Jin-Woo Park (D) ${ }^{1}$, Emad Oveisi (1) ${ }^{6}$, \\ Satyawan Nagane7, Aditya Sadhanala7,8,16, Lijun Zhang (1) 4, Jin Jung Kweon', Sung Keun Lee (10) 9,10, \\ Hoichang Yang ${ }^{2}$, Hyun Myung Jang (1) ${ }^{11}$, Richard H. Friend (iD ${ }^{7}$, Kian Ping Loh (1) ${ }^{3}$, \\ Mohammad Khaja Nazeeruddin (10 ${ }^{12}$, Nam-Gyu Park (D) ${ }^{13}$ \& Tae-Woo Lee (iD $1,14,15 \bowtie$
}

Perovskite light-emitting diodes (PeLEDs) based on three-dimensional (3D) polycrystalline perovskites suffer from ion migration, which causes overshoot of luminance over time during operation and reduces its operational lifetime. Here, we demonstrate 3D/2D hybrid PeLEDs with extremely reduced luminance overshoot and 21 times longer operational lifetime than 3D PeLEDs. The luminance overshoot ratio of 3D/2D hybrid PeLED is only $7.4 \%$ which is greatly lower than that of 3D PeLED (150.4\%). The 3D/2D hybrid perovskite is obtained by adding a small amount of neutral benzylamine to methylammonium lead bromide, which induces a proton transfer from methylammonium to benzylamine and enables crystallization of 2D perovskite without destroying the 3D phase. Benzylammonium in the perovskite lattice suppresses formation of deep-trap states and ion migration, thereby enhances both operating stability and luminous efficiency based on its retardation effect in reorientation.

\footnotetext{
${ }^{1}$ Department of Materials Science and Engineering, Seoul National University, Seoul 08826, Republic of Korea. ${ }^{2}$ Department of Chemical Engineering, Inha University, Incheon 22212, Republic of Korea. ${ }^{3}$ Department of Chemistry, National University of Singapore, Singapore 117543 , Singapore. ${ }^{4}$ State Key Laboratory of Integrated Optoelectronics, Key Laboratory of Automobile Materials of MOE and College of Materials Science and Engineering, Jilin University, Changchun 130012, China. ${ }^{5}$ Research Center for Materials Analysis, Korea Basic Science Institute (KBSI), Daejeon 34133, Republic of Korea.

${ }^{6}$ Interdisciplinary Centre for Electron Microscopy (CIME), École Polytechnique Fédérale de Lausanne (EPFL), Lausanne CH-1951, Switzerland. ${ }^{7}$ Cavendish Laboratory, University of Cambridge, JJ Thomson Avenue, Cambridge CB3 OHE, UK. ${ }^{8}$ Clarendon Laboratory, Department of Physics, University of Oxford, Parks Road, Oxford OX1 3PU, UK. ${ }^{9}$ School of Earth and Environmental Sciences, Seoul National University, Seoul 08826, Republic of Korea. ${ }^{10}$ Institute of Applied Physics, Seoul National University, Seoul 08826, Republic of Korea. ${ }^{11}$ Department of Materials Science and Engineering, Pohang University of Science and Technology, Pohang 37673, Republic of Korea. ${ }^{12}$ Group for Molecular Engineering of Function Materials, Institute of Chemical Sciences and Engineering, École Polytechnique Fédérale de Lausanne (EPFL), Sion CH-1951, Switzerland. ${ }^{13}$ Department of Materials Science and Engineering, Sungkyunkwan University, Suwon 16419, Republic of Korea. ${ }^{14}$ School of Chemical and Biological Engineering, Seoul National University, Seoul 08826, Republic of Korea. ${ }^{15}$ Institute of Engineering Research, Research Institute of Advanced Materials, Nano Systems Institute (NSI), Seoul National University, Seoul 08826, Republic of Korea. ${ }^{16}$ Present address: Centre for Nano Science and Engineering, Indian Institute of Science, Bangalore 560012, India. ${ }^{17}$ These authors contributed equally: Hobeom Kim, Joo Sung Kim, Jung-Min Heo. ${ }^{凶}$ email: twlees@snu.ac.kr
} 
$\mathrm{n}$ the early days of the research on perovskites light-emitting diodes (PeLEDs), three-dimensional (3D) polycrystalline perovskites have been employed as a light-emitter based on the advantages such as high colour purity, facile tuning of emission colour, low material cost and low-temperature processability ${ }^{1-3}$. Extensive research has contributed to rapid increase in luminous efficiency of PeLEDs by various approaches, such as introduction of nanocrystal pinning (NCP) process, management of compositional distribution, incorporation of polymer into perovskite and an enhancement in light outcoupling ${ }^{3-6}$. However, 3D polycrystalline perovskite emitters have a fundamental limitation in efficient radiative recombination because of the high density of trap states which originates primarily from ionic defects and their migration degrades the operating lifetime of PeLEDs; this problem must be overcome before they can be practically used for lighting and displays.

There have been many reports on PeLEDs regarding overshoot of luminance during the initial stage of operation ${ }^{4,6-8}$. However, the mechanism of this phenomenon has not been identified. The ion migration can much negatively influence on device stability because the PeLEDs operate under much higher applied electric field compared with perovskite solar cells. Therefore, to improve the device lifetime of PeLEDs, ion migration must be suppressed $^{7,9-12}$. Especially, the ion migration mostly occurs on grain boundaries (GBs), thus blocking possible migration pathways along the GB can be an effective strategy to suppress it ${ }^{13}$.

In this study, we develop a proton-transfer-induced $3 \mathrm{D} / 2 \mathrm{D}$ hybrid perovskite emitter that can be an ideal configuration to suppress the ion migration and significantly reduce the overshoot of luminance with improved operational stability in PeLEDs during the initial stage. In a conventional way to prepare lowdimensional layered perovskites, an ammonium halide salt with a bulky backbone (e.g. phenethylammonium halide (PEAX), or $n$-butylammonium halide (n-BAX)) and the salts of a $3 \mathrm{D}$ perovskite (e.g. methylammonium halide (MAX) and lead halide $\left(\mathrm{PbX}_{2}\right)$ ) have been dissolved together in a solvent or a mixed solvent $^{8,14-19}$. Here, unlike the conventional way, we incorporate a liquid-form neutral reagent, benzylamine (BnA) into the methylammonium lead bromide $\left(\mathrm{MAPbBr}_{3}\right)$ precursor to form $3 \mathrm{D} / 2 \mathrm{D}$ hybrid perovskites ${ }^{20}$. Therefore, the synthetic procedure which requires strong hydrohalic acid reagents to obtain the organic

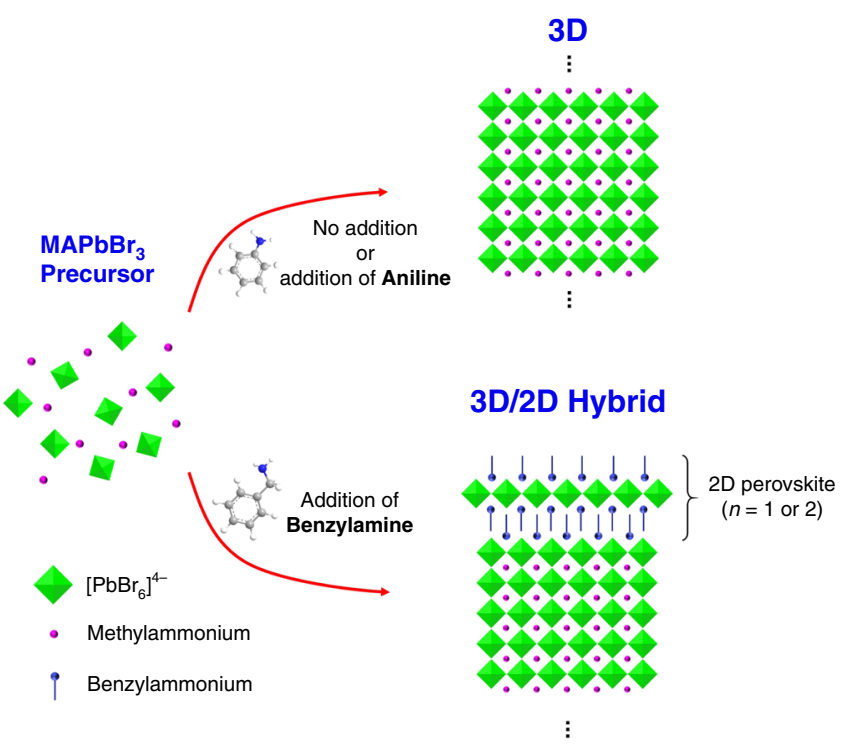

Fig. 1 Principal scheme of our study. The neutral reagent, benzylamine $(\mathrm{BnA})$ leads to the formation of 3D/2D hybrid perovskites while aniline (ANI) does not induce the formation of $2 \mathrm{D}$ perovskites. ammonium halide salt for $2 \mathrm{D}$ perovskites becomes unnecessary. To compare with $\mathrm{BnA}$, we use aniline (ANI) as it has the same molecular structure except for a methylene bridge between benzene ring and amine. ${ }^{1} \mathrm{H}$ nuclear magnetic resonance (NMR) spectroscopy revealed that the strongly basic BnA participates in the crystallization of $2 \mathrm{D}$ perovskite by being transformed into $\mathrm{BnA}^{+}$by proton-transfer from $\mathrm{MA}^{+}$in the precursor. Grazingincident $\mathrm{x}$-ray diffraction (GIXD) technique confirmed the formation of $2 \mathrm{D} \mathrm{BnA} \mathrm{BbBr}_{4}$, dominantly with $n=1$, while retaining the $3 \mathrm{D} \mathrm{MAPbBr}_{3}$ phase. In contrast, ANI which has a similar molecular structure with $\mathrm{BnA}$ does not work as a reagent because it is weakly basic, so it cannot induce the formation of additional $2 \mathrm{D}$ perovskite. The $3 \mathrm{D} / 2 \mathrm{D}$ hybrid perovskite film showed a significant improvement in photoluminescence (PL) characteristics compared to the $3 \mathrm{D}$ perovskite film because the $2 \mathrm{D}$ perovskite can passivate deep trap sites, as well as shallow traps in 3D perovskite, and thereby lead to efficient radiative recombination. Therefore, PeLEDs with the $3 \mathrm{D} / 2 \mathrm{D}$ hybrid perovskite emitter had highly improved electroluminescence (EL) efficiency. Furthermore, the $3 \mathrm{D} / 2 \mathrm{D}$ hybrid PeLEDs showed a dramatic enhancement in the long-term stability owing to the suppressed ion migration, which led to an extremely reduced initial luminance overshoot ratio as $7.4 \%$. In contrast, the $3 \mathrm{D}$ PeLEDs which suffer from ion migration showed a steep increase in initial luminance with the overshoot ratio as $150.4 \%$. The $3 \mathrm{D} / 2 \mathrm{D}$ hybrid PeLED had more than 21 times longer operational lifetime $\left(T_{40}=810 \mathrm{~min}\right)$ than 3D PeLEDs $\left(T_{40}=38 \mathrm{~min}\right)$. We cohesively link the PL improvements and the suppression of ion migration to the retardation effect of $\mathrm{BnA}^{+}$ reorientation in the perovskite lattice.

\section{Results}

Protonation of $\mathrm{BnA}$ enabling 2D perovskite formation. The incorporation of $\mathrm{BnA}$ into $\mathrm{MAPbBr}_{3}$ precursor leads to the formation of $3 \mathrm{D} / 2 \mathrm{D}$ hybrid dimensional perovskite in which the formation of $2 \mathrm{D}$ perovskite does not degrade the existing 3D phase so that $3 \mathrm{D}$ and $2 \mathrm{D}$ perovskites coexist (Fig. 1). In contrast, the addition of ANI does not make the structural change maintaining the 3D phase of perovskite as the case without an additive. We performed ${ }^{1} \mathrm{H}$ NMR spectroscopy to understand the underlying chemistry in $\mathrm{MAPbBr}_{3}$ precursor according to the addition of $2.4 \mathrm{~mol} \%$ of ANI or $\mathrm{BnA}$ compared to $\mathrm{MAPbBr}_{3}$ without the addition before the crystallization of perovskites occurs (Fig. 2a). The precursors were dissolved in dimethyl sulfoxide- $d_{6}$ (DMSO$d_{6}$ ) as the same condition of the films for actual PeLEDs which we will discuss in the following sections. The spectrum of pristine $\mathrm{MAPbBr}_{3}$ precursor showed two dominant proton signals, one at $\delta=7.44 \mathrm{ppm}$ that represents the ammonium $\left(-\mathrm{NH}_{3}{ }^{+}\right)$of $\mathrm{MA}^{+}$, and the other at $2.32 \mathrm{ppm}$ that represents its methyl $\left(-\mathrm{CH}_{3}\right)$ group. The addition of $\mathrm{BnA}$ to the precursor of $\mathrm{MAPbBr}_{3}$ caused an upfield shift of the proton signal of the ammonium group to $\delta=7.31 \mathrm{ppm}$. The proton signal of the ammonium group progressively showed further upfield shift as the amount of BnA addition increased; the signal reached $6.33 \mathrm{ppm}$ at $50 \mathrm{~mol} \% \mathrm{BnA}$ and $5.67 \mathrm{ppm}$ at $100 \mathrm{~mol} \% \mathrm{BnA}$ (Fig. 2b). These changes can be attributed to the strong shielding of the ammonium moiety of $\mathrm{MA}^{+}$by $\mathrm{BnA}$ reagent. In contrast, the addition of ANI to the $\mathrm{MAPbBr}_{3}$ precursor did not cause any shift. Even high concentrations of ANI did not cause a noticeable chemical shift of the proton signal of MA (Fig. 2c); this lack of effect indicates that the solution with ANI remained as a mixture without causing a chemical reaction even though ANI has the same molecular structure as $\mathrm{BnA}$ except the methylene bridge $\left(-\mathrm{CH}_{2}\right)$ between the amine and the benzene ring. The integration ratio $(I)$ of proton signals $\left(H_{\text {ammonium }} / H_{\text {alkyl }}\right)$ increased from 1 for pristine $\mathrm{MAPbBr}_{3}$ to 1.81 for $\mathrm{MAPbBr}_{3}$ with $100 \mathrm{~mol} \%$ of $\mathrm{BnA}$ 
a

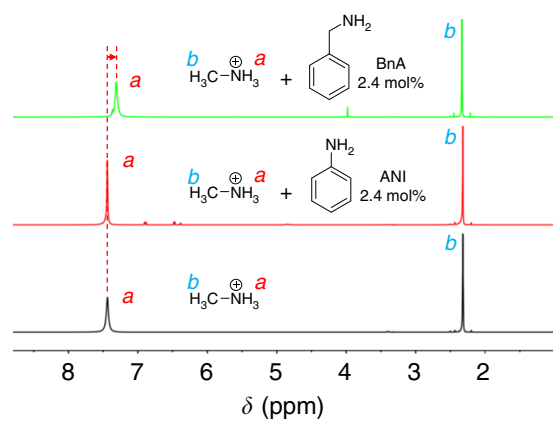

b

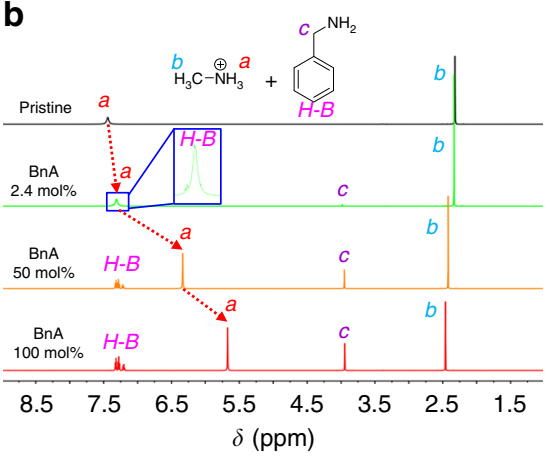

C

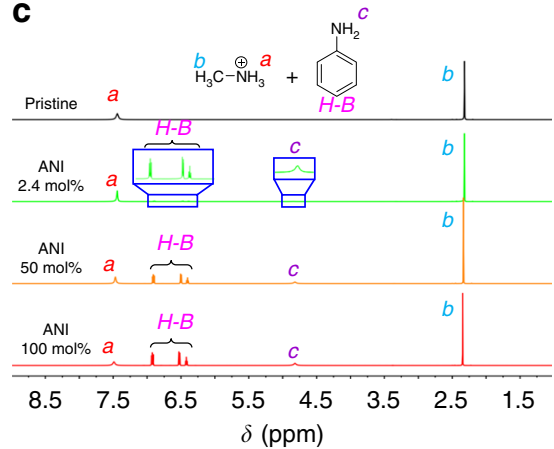

d
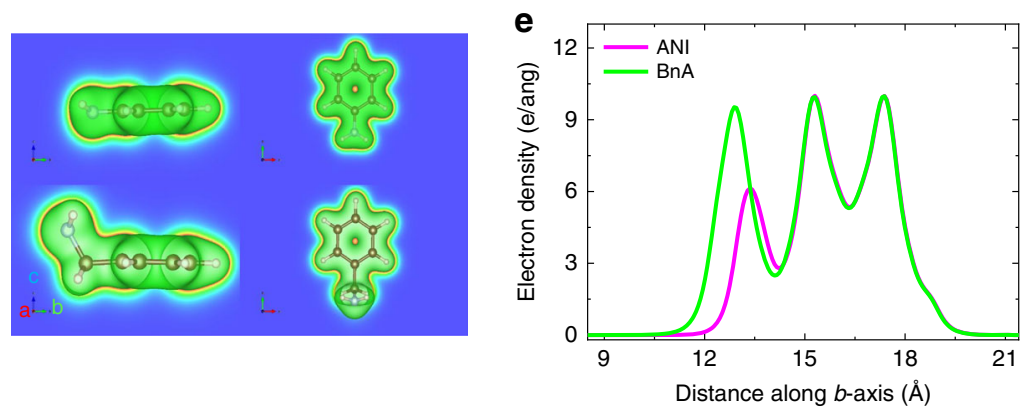

f

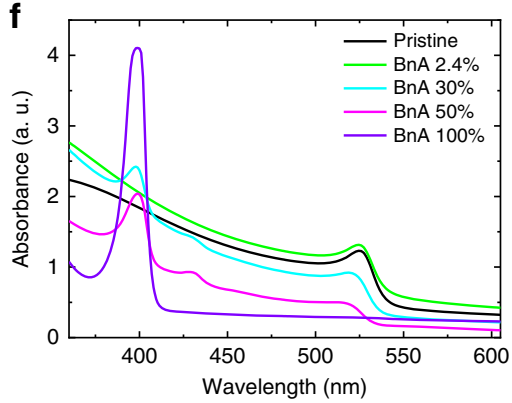

g

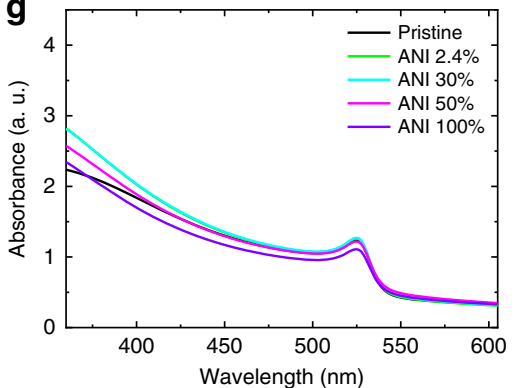

h<smiles>[1H][NH+](C)[18OH]</smiles>

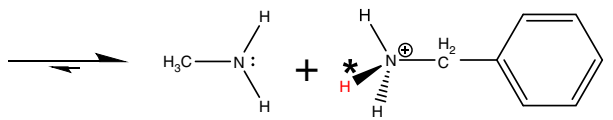

Fig. 2 Proton-transfer-induced 2D perovskite formation with BnA. a ${ }^{1} \mathrm{H} N M R$ spectra of $M A P b B r_{3}$ precursor solutions in $\mathrm{DMSO} \mathrm{d}_{6}$ according after addition of additives. Pristine $\mathrm{MAPbBr}_{3}$ (black), $\mathrm{MAPbBr}_{3}$ with 2.4 mol\% of $\mathrm{ANI}$ (black), and $\mathrm{MAPbBr}_{3}$ with 2.4 mol\% of $\mathrm{BnA}$ (green). b, c ${ }^{1} \mathrm{H} \mathrm{NMR}$ spectra according to the different amount of $\mathrm{BnA}$ and $\mathrm{ANI}$ addition. $\mathbf{d}$ Electron density contour plots of BnA (bottom) and ANI (top) with an iso-surface of 0.03 e $\AA^{-3}$. e Integrated electron density profile for $\mathrm{BnA}$ (green) and $\mathrm{ANI}$ (red) along with $b$-axis in $\mathbf{d} . \mathbf{f}, \mathbf{g} \mathrm{Absorption}$ spectra of $\mathrm{MAPbBr}{ }_{3}$ thin film according to the addition of $\mathrm{BnA}$ and $\mathrm{ANI}$. $\mathbf{h}$ Proton-transfer reaction between MA and $\mathrm{BnA}$.

(Supplementary Fig. 1 and Supplementary Table 1). This change indicates the presence of a strong interaction that represents an increased hydrogen bond strength by sharing a proton between $\mathrm{BnA}$ and $\mathrm{MA}^{+}$. In contrast, $\mathrm{MAPbBr}_{3}$ with $100 \mathrm{~mol} \%$ ANI had I around 1 implying that ANI does not interact with $\mathrm{MA}^{+}$.

These different effects of BnA and ANI on the chemical behaviour can be explained by the Brønsted-Lowry acid-base reaction. BnA is a strong base, so a proton from the ammonium group of $\mathrm{MA}^{+}$which is a weak acid, can be transferred to nitrogen of $\mathrm{BnA}$ to yield $\mathrm{BnA}^{+}$. In contrast, ANI is a weak base, so it cannot extract a proton from $\mathrm{MA}^{+}$. To learn the cause of this difference, we calculated the electron densities of $\mathrm{BnA}$ and ANI with the first-principles density functional theory (DFT). The resultant contour plots of the electron density of each molecule and its integrated profiles along the $b$-axis direction are presented (Fig. 2d, e). The calculated results indicate that BnA has a much higher electron density on the terminal amine moiety than ANI because the methylene bridge on BnA can donate more electrons to the more electronegative nitrogen and localise the electrons on the nitrogen leading to an inductive effect. ANI, in contrast, has electrons that are delocalized between the nitrogen and the benzene ring governed by a resonance effect, which makes ANI much less basic than BnA. As a consequence of these differences, the protonation tendency from $\mathrm{MA}^{+}$to $\mathrm{BnA}$ is $5.5 \times 10^{4}$ times stronger than from $\mathrm{MA}^{+}$to ANI (Supplementary Note 1).

The absorption characteristics of the solid-state perovskite films from the corresponding precursors support this result (Fig. 2f, g): the excitonic peak of the $\mathrm{MAPbBr}_{3}$ absorption spectrum at $525 \mathrm{~nm}$ changes significantly according to the amount of BnA added, but does not shift with the addition of ANI regardless of the amount. For example, the addition of $30 \mathrm{~mol} \% \mathrm{BnA}$ resulted in 

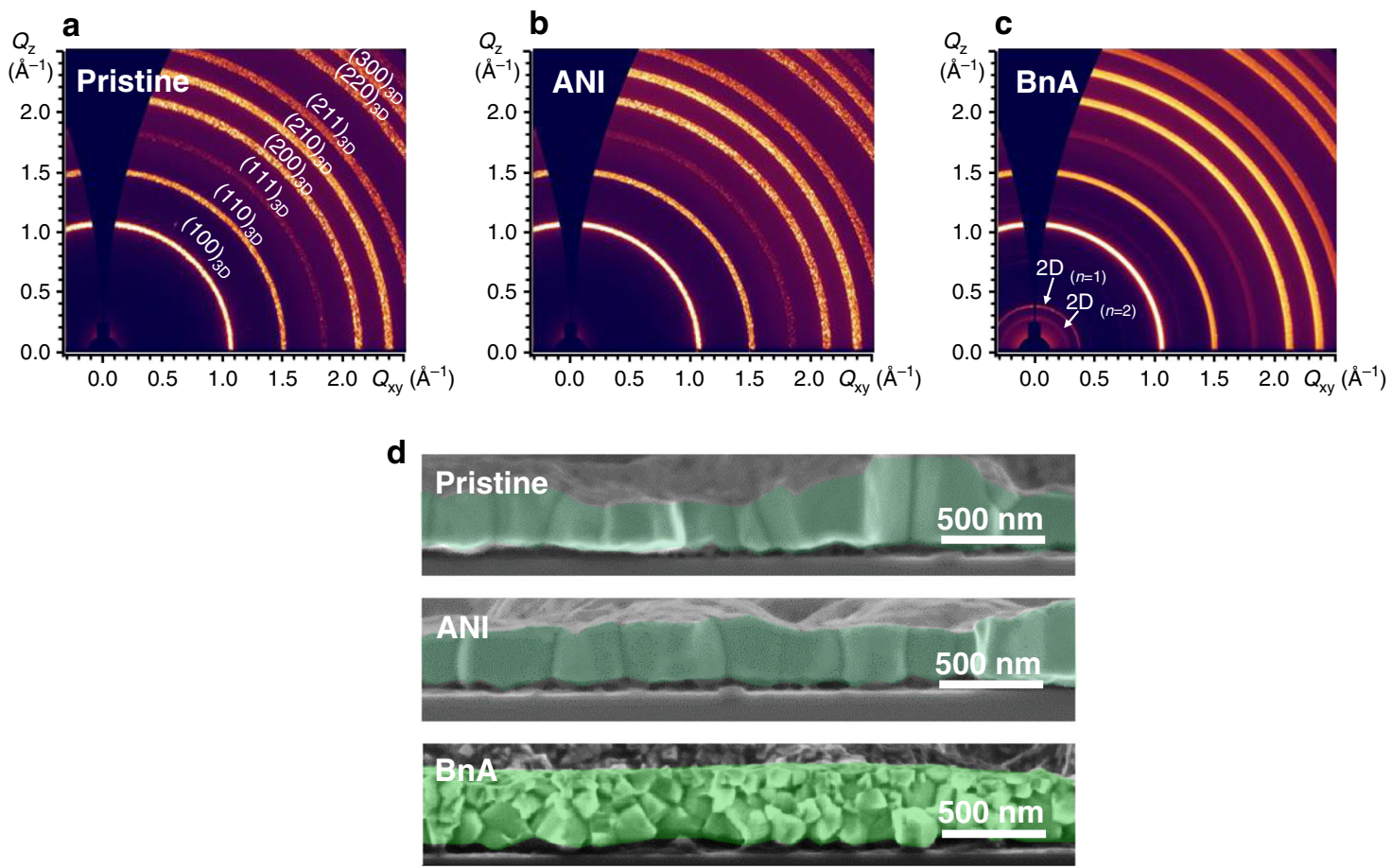

Fig. 3 Crystal structure and morphology of $\mathbf{M A P b B r}_{\mathbf{3}}$ according to the addition of $\mathbf{A N I}$ or $\mathbf{B n A}$. a-c $2 \mathrm{D}$ GIXD patterns of pristine $M A P b B r_{3}, M A P b B r_{3}$ with $2.4 \mathrm{~mol} \% \mathrm{ANI}$, and 2.4 mol\% BnA. d Cross-sectional SEM images of pristine $\mathrm{MAPbBr}_{3}$ (top), $\mathrm{MAPbBr}_{3}$ with 2.4 mol\% ANI (middle), and 2.4 mol\% BnA (bottom).

new excitonic peaks at 399 and $430 \mathrm{~nm}$, which can be assigned to a phase with $n=1$ and 2, respectively, indicating the formation of $2 \mathrm{D}$ perovskites ${ }^{21-24}$. Interestingly, the film obtained using a precursor with $100 \mathrm{~mol} \%$ of $\mathrm{BnA}$ showed the spectrum of a typical $2 \mathrm{D}$ perovskite film that has an excitonic peak at $399 \mathrm{~nm}$; this peak is the result of the strong proton-withdrawing tendency of $\mathrm{BnA}$ which takes protons from $\mathrm{MA}^{+}$and participates in the formation of $2 \mathrm{D}$ perovskite (Fig. 2h). Furthermore, we predicted the importance of the protonation of $\mathrm{BnA}$ by performing firstprinciples molecular dynamics (MD) simulations in which perovskite lattice including the protonated $\mathrm{BnA}\left(\mathrm{BnA}^{+}\right)$showed much greater structural stability than that with neutral BnA. (Supplementary Fig. 2). Also, we performed the solid-state magicangle-spinning (MAS) NMR spectroscopy to gain an atomic-level insight about the formation of crystalline perovskite (Supplementary Fig. 3). The result confirmed that the protonated $\mathrm{BnA}$ composed the solid-state crystalline perovskite while ANI did not participate in the formation of perovskite.

Crystal structure and configuration of perovskites. To examine the detail of perovskite crystal structures and dimensionality, we performed grazing-incidence X-ray diffraction (GIXD) on the perovskite films. The results confirmed that BnA contributed to the formation of $2 \mathrm{D}$ perovskite but that ANI did not; this result is consistent with the conclusions drawn from the ${ }^{1} \mathrm{H}$ NMR analysis and optical absorption spectra. The films of 3D pristine $\mathrm{MAPbBr}_{3}$ and $\mathrm{MAPbBr}_{3}$ with $2.4 \mathrm{~mol} \%$ ANI showed similar diffraction patterns; this result indicates that the ANI does not influence the perovskite crystal structure. In contrast, the addition of $2.4 \mathrm{~mol} \%$ $\mathrm{BnA}$ led to an appearance of additional peaks at low angles; the most dominant one was at $2 \theta=5.32^{\circ}$ (Supplementary Fig. 4). The newly appeared peak can be assigned to a $2 \mathrm{D}$ perovskite with the lattice plane spacing of $16.58 \AA$ according to Bragg's equation, which corresponds to $\mathrm{BnA}_{2} \mathrm{PbBr}_{4}$ with $n=1$ (Table S2). The information about the crystal structure of perovskite also can be shown in 2D GIXD patterns (Fig. 3a-c). It is clear that the $\mathrm{MAPbBr}_{3}$ film with $2.4 \mathrm{~mol} \% \mathrm{BnA}$ had an additional X-ray reflection ring at scattering vector $Q=0.38 \AA^{-1}$; this is a consequence of the $2 \mathrm{D}$ phase. In contrast, the $\mathrm{MAPbBr}_{3}$ with $2.4 \mathrm{~mol} \% \mathrm{ANI}$ had the same pattern as the pristine film of the $3 \mathrm{D}$ phase. It is worth noting that $\mathrm{MAPbBr}_{3}$ with $\mathrm{BnA}$ retained the diffraction patterns of $3 \mathrm{D}$ phase; i.e., $3 \mathrm{D} \mathrm{MAPbBr}_{3}$ and $2 \mathrm{D}$ $\mathrm{BnA}_{2} \mathrm{PbBr}_{4}$ coexisted in the film, which can be called $3 \mathrm{D} / 2 \mathrm{D}$ hybrid perovskite. Ring-shaped diffraction patterns with a uniform intensity indicate a random orientation and can be attributed to a NCP process that renders grains small by rapid crystallization of perovskite ${ }^{25}$; such small grains can induce an efficient radiative recombination ${ }^{3,26-28}$. An increase in the ratio of $\mathrm{BnA}$ to $30 \mathrm{~mol} \%$ increased the intensity of the signal of the $2 \mathrm{D}$ perovskite phase, whereas the increase in the amount of ANI addition did not cause the formation of any other phase while maintaining 3D phase (Supplementary Fig. 4).

The addition of BnA caused an apparent change in the film morphology of perovskites. The films of pristine $\mathrm{MAPbBr}_{3}$ and $\mathrm{MAPbBr}_{3}$ with $2.4 \mathrm{~mol} \%$ ANI had a similar columnar grain structure, whereas 3D/2D hybrid perovskite film with $2.4 \mathrm{~mol} \%$ $\mathrm{BnA}$ had granular grains with a smaller size (Fig. $3 \mathrm{~d}$ ). This is because the growth of $2 \mathrm{D}$ perovskite can decrease the growth rate of $3 \mathrm{D}$ perovskite leading to the formation of a smaller granular type of grains, which can be beneficial to efficient exciton confinement within a grain ${ }^{3,26}$. The morphology of $3 \mathrm{D} / 2 \mathrm{D}$ hybrid perovskite films with $\mathrm{BnA}$ showed a strong dependence on the amount of BnA (Supplementary Fig. 5). However, the addition of even a large amount of ANI had little influence on the morphology of $\mathrm{MAPbBr}_{3}$ film.

Improvements in PL characteristics and trap passivation. We investigated PL characteristics of the $3 \mathrm{D}$ and the $3 \mathrm{D} / 2 \mathrm{D}$ hybrid perovskite films. The perovskite films were prepared on a glass substrate/buffer hole-injection layer (Buf-HIL), which consists of 

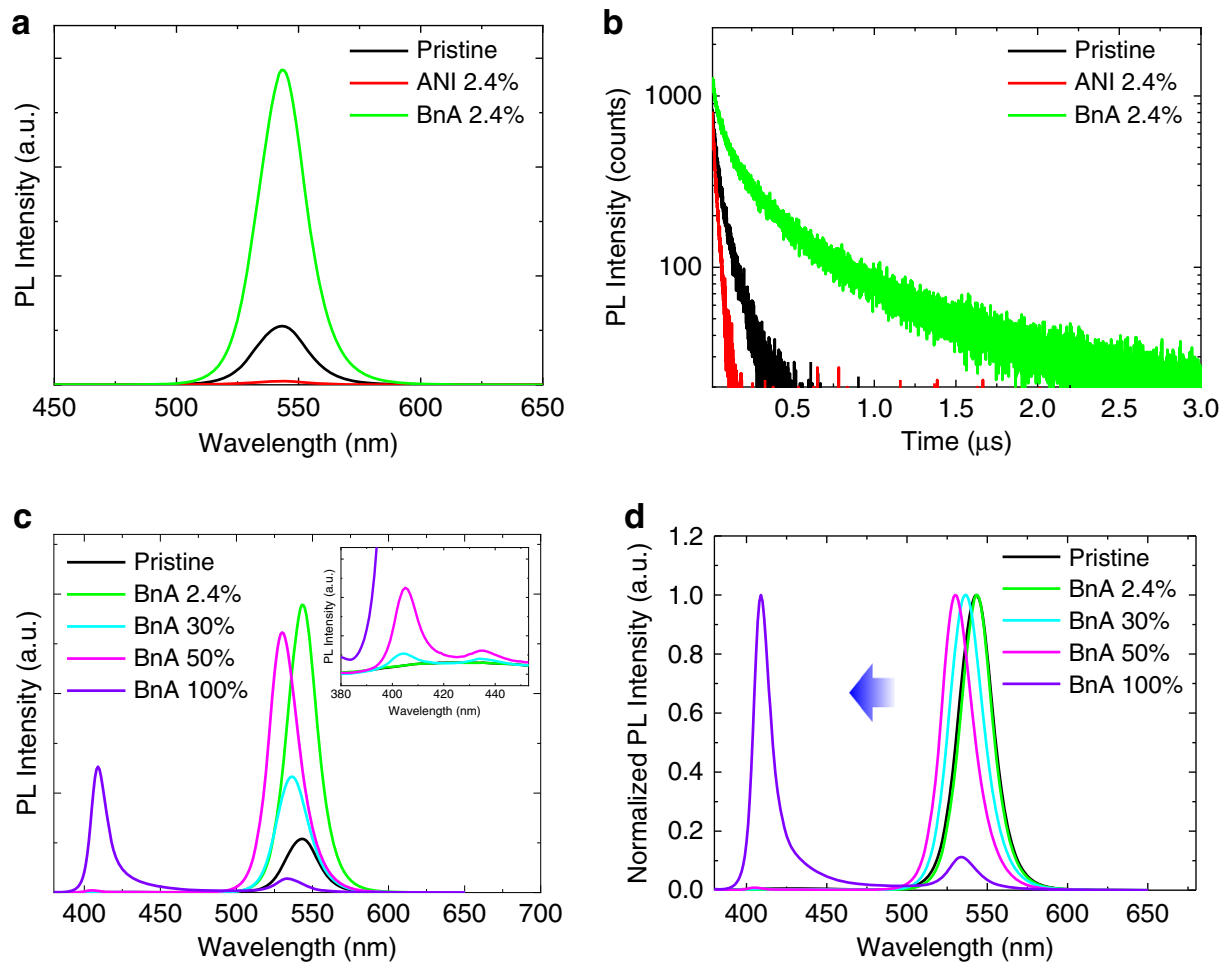

Fig. 4 PL characteristics of perovskites and the trap passivation. a PL spectrum of pristine MAPbBr ${ }_{3}$ film (black), film with 2.4 mol\% of $A N I$ (red), and $2.4 \mathrm{~mol} \%$ of $\mathrm{BnA}$ (green). b PL lifetime curves of pristine $\mathrm{MAPbBr}_{3}$ film (black), film with $2.4 \mathrm{~mol} \%$ of $\mathrm{ANI}$ (red), and $2.4 \mathrm{~mol} \%$ of $\mathrm{BnA}$ (green). c PL spectra of $\mathrm{MAPbBr}_{3}$ film with different amount of $\mathrm{BnA}$ and magnified PL spectra at low wavelength region (inset). $\mathbf{d}$ Normalized $\mathrm{PL}$ spectra of $M A P b B r_{3}$ film with different amount of BnA.

poly(3,4-ethylenedioxythiophene) polystyrene sulfonate (PEDOT: PSS) and perfluorinated ionomer. In steady-state PL measurement, the 3D/2D hybrid perovskite film with $2.4 \mathrm{~mol} \% \mathrm{BnA}$ showed a much higher PL intensity than the $3 \mathrm{D}$ perovskite film, whereas the addition of $2.4 \mathrm{~mol} \%$ ANI caused significant PL quenching (Fig. 4a). Also, the transient PL measurement of the films resulted in the same trend as in the steady-state PL (Fig. 4b). The PL average lifetime $\tau_{\text {Ave }}$ was more than four times longer in $3 \mathrm{D} / 2 \mathrm{D}$ hybrid perovskite film $\left(\tau_{\mathrm{Ave}}=254.4 \mathrm{~ns}\right)$ than in pristine $3 \mathrm{D}$ film $\left(\tau_{\text {Ave }}=60.94 \mathrm{~ns}\right)$, but the film with ANI showed considerably shortened PL lifetime $\left(\tau_{\text {Ave }}=24.71 \mathrm{~ns}\right)$. We summarized the detailed decay parameters based on the bi-exponential decay function in Supplementary Table 3. We realized that 2.4 $\mathrm{mol} \% \mathrm{BnA}$ did not cause a shift in the peak position compared to the $\mathrm{PL}$ of $3 \mathrm{D} \mathrm{MAPbBr} 3$ at $543 \mathrm{~nm}$, or cause the formation of 2D perovskite PL emission peaks. These observations indicate that the radiative recombination occurs only in the $3 \mathrm{D}$ perovskite rather than in the $2 \mathrm{D}$ perovskite, which can be attributed to the energy cascade that results from the difference in the dimensionality between perovskite phases in the $3 \mathrm{D} / 2 \mathrm{D}$ hybrid structure (Fig. 4c, d) ${ }^{16,29}$. Only the addition of the larger amount of BnA caused a blue-shift of PL emission (to $537 \mathrm{~nm}$ for $30 \mathrm{~mol} \%$ and to $530 \mathrm{~nm}$ for $50 \mathrm{~mol} \%$ ), accompanied by the appearance of 2D perovskite PL emissions at 405 and $435 \mathrm{~nm}$, which can be assigned to $n=1$ and 2, respectively. The further addition of BnA up to $100 \mathrm{~mol} \%$ which corresponds to the same amount as MA, caused a dramatic increase in the intensity of PL emission at 409 $\mathrm{nm}$ from $2 \mathrm{D}$ perovskite. This shift can be more clearly seen in normalized PL spectra (Fig. 4d). We also measured the absolute PL quantum efficiency (PLQE) which showed a similar trend to the steady-state PL intensity according to the amount of BnA (Supplementary Fig. 6). On the other hand, increasing the amount of ANI only decreased the PL intensity, and did not shift the emission wavelength, which can be attributed to intensive non-radiative recombination due to the electron deficiency of ANI (Supplementary Fig. 7) ${ }^{30}$.

Also, we showed that alignment of energy bands contributes to the improvement in PL properties of the $3 \mathrm{D} / 2 \mathrm{D}$ hybrid perovskite film based on ultraviolet photoemission spectroscopy (UPS) analysis (Supplementary Figs. 8, 9 and Supplementary Note 2). We further investigated the carrier confinement effect in the perovskites by performing the excitation density-dependent PL measurement at room temperature (Supplementary Fig. 10). In the excitation density range of $10^{15}-10^{18} \mathrm{~cm}^{-3}$ which corresponds to the operation regime of $\mathrm{LEDs}^{31}$, the integrate $\mathrm{PL}\left(\mathrm{PL}_{\mathrm{int}}\right)$ follows a power-law dependence on the excitation density, $n_{\mathrm{ex}}$ as $\mathrm{PL}_{\mathrm{int}} \propto n_{\mathrm{ex}}{ }^{k}$ where the $3 \mathrm{D}$ perovskite had $k=1.30$ indicating the co-existence of excitons (monomolecular emission) and free carriers (bimolecular emission), while the $3 \mathrm{D} / 2 \mathrm{D}$ hybrid perovskite film showed $k=1.08$ indicating the predominant excitonic character by monomolecular recombination, which can be attributed to the effective carrier confinement ${ }^{32}$.

To deeply understand the trap passivation effect by using $3 \mathrm{D} /$ 2D hybrid perovskite, we investigated temperature-dependent steady-state PL characteristics of the perovskite films from 70 to $300 \mathrm{~K}$. The $3 \mathrm{D}$ perovskite film showed two distinct PL emission peaks at low temperatures below $130 \mathrm{~K}$ (Fig. 5a). While the sharp PL peaks with higher energy can arise from band-edge (BE) emission, the broad PL band with lower energy can be attributed to shallow trap states induced by structural disorder in the orthorhombic phase of $\mathrm{MAPbBr}_{3}{ }^{33,34}$. In this temperature regime, the ratio of integrated $\mathrm{PL}$ intensity of $\mathrm{BE}$ versus the shallow trap-mediated PL emission (TE) was increasing as temperature increased, which implies that thermally assisted 

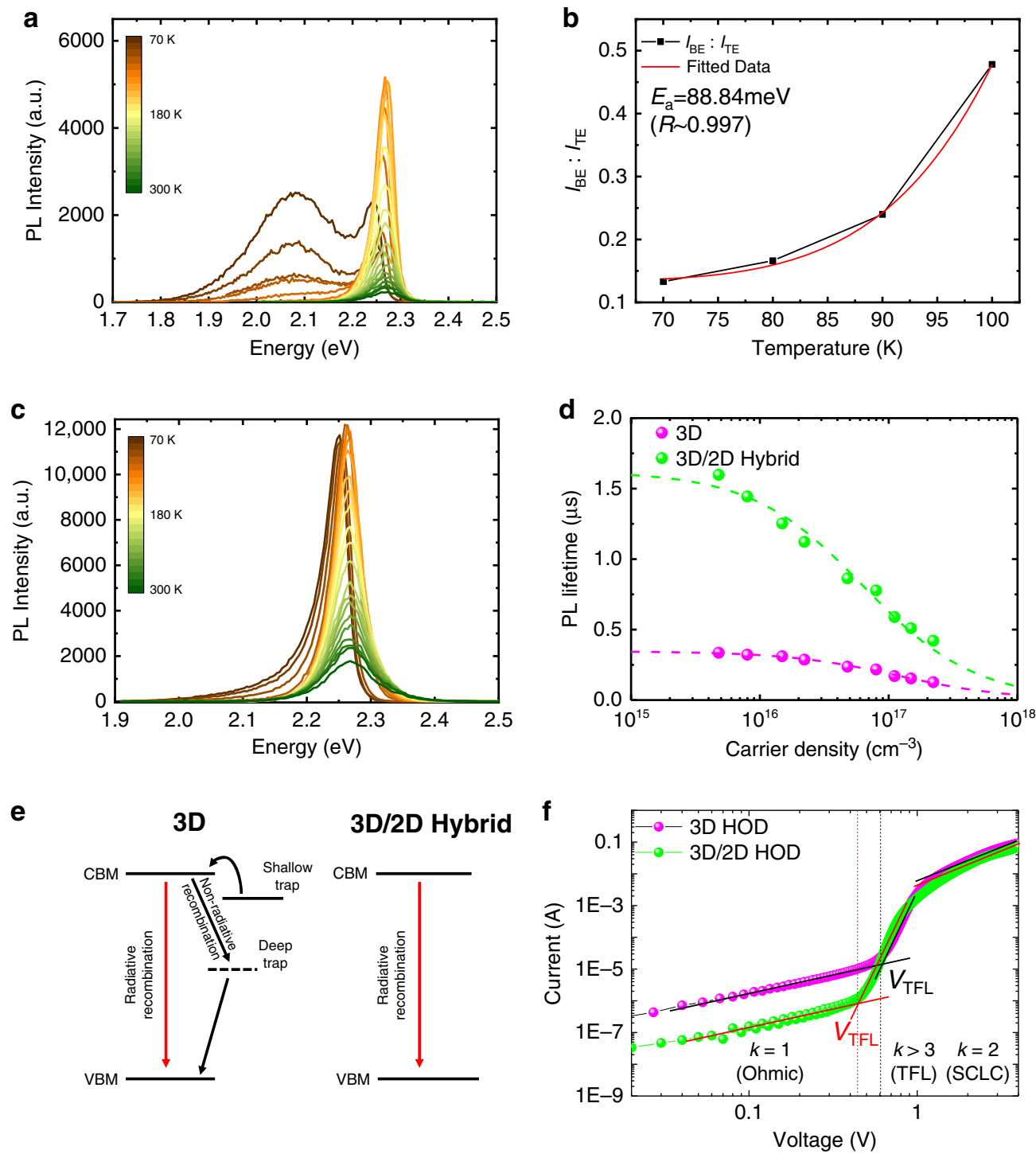

Fig. 5 Photo-physical analyses to investigate recombination kinetics and trap states of perovskites. a Temperature-dependent $P L$ of $3 D$ perovskite and b Ratio between integrated PL of band emission and trap-mediated emission of 3D perovskite as a function of temperature and its fitted curve according to Boltzmann distribution function. c Temperature-dependent PL of 3D/2D hybrid perovskite. $\mathbf{d}$ Excited charge carrier density-dependent PL lifetimes of the perovskites. e Schematic of charge carrier recombination dynamics of the perovskites. $\mathbf{f} I-V$ curves of hole-only devices with 3D perovskite and 3D/2D hybrid perovskite.

de-trapping of charge carriers occurs (Fig. $5 b$ and Supplementary Fig. 11). To estimate the value of the activation energy to de-trap the charge carriers from the shallow traps, the curve was fitted by the Boltzmann distribution function:

$$
y=y_{0} \mathrm{e}^{\left(-\frac{E_{\mathrm{a}}}{k_{\mathrm{B}} T}\right)}
$$

where $k_{\mathrm{B}}$ is the Boltzmann constant and $T$ is temperature and $E_{\mathrm{a}}$ is the activation energy to de-trap charge carriers, which resulted in $88.84 \mathrm{meV}$. In contrast, the $3 \mathrm{D} / 2 \mathrm{D}$ hybrid perovskite film only showed a single PL peak of BE without the TE in the whole range of temperature (Fig. $5 \mathrm{c}$ ). This indicates that the incorporation of $2 \mathrm{D}$ perovskites effectively passivates the shallow trap states even in the orthorhombic phase.

Furthermore, the excitation density dependence of the PL lifetimes revealed defect-related recombination kinetics of the perovskites (Fig. 5d). We controlled the excited charge carrier density of the perovskite films from $4.8 \times 10^{15}$ to $2.2 \times 10^{17} \mathrm{~cm}^{-3}$ (Supplementary Fig. 12). We used a rate equation model where the decay of the excited charge carrier density $n_{0}$ can be explained regarding radiative and non-radiative recombination, which can be written as

$$
\tau_{\mathrm{PL}}=\left(A+B n_{0}\right)^{-1}
$$

where $A$ is the non-radiative recombination rate coefficient, and $B$ is the radiative recombination rate coefficient. We found that the 3D/2D hybrid perovskite film exhibited a significantly lower $A=$ $6.16 \times 10^{5} \mathrm{~s}^{-1}$ than that of $3 \mathrm{D}$ perovskite $\left(A=2.96 \times 10^{6} \mathrm{~s}^{-1}\right)$. This indicates that the $3 \mathrm{D}$ perovskite has a high density of nonradiative Shockley-Read-Hall recombination centre which can be associated with certain kinds of ionic defects in the lattice despite a high degree of defect tolerance of perovskite ${ }^{35}$. In contrast, the incorporation of $2 \mathrm{D}$ perovskite into $3 \mathrm{D}$ perovskite can restrict the defect formation and migration leading to such lowered nonradiative recombination rate coefficient. This is due to the presence of $\mathrm{BnA}^{+}$which has a lower rotational degree of freedom than $\mathrm{MA}^{+36}$, which will be discussed in detail in the following section. Meanwhile, the $3 \mathrm{D}$ perovskite and the $3 \mathrm{D} / 2 \mathrm{D}$ hybrid 
perovskite showed a similar radiative recombination rate coefficient, $B$ as $2.30 \times 10^{-11}$ and $1.08 \times 10^{-11} \mathrm{~cm}^{3} \mathrm{~s}^{-1}$, respectively. This result well matches with the explanation that the shallow traps mostly disappeared in both perovskites. On the basis of the results of the comprehensive PL analyses, we depicted a schematic of the charge recombination dynamics of 3D and 3D/ $2 \mathrm{D}$ hybrid perovskites (Fig. $5 \mathrm{e}$ ). In the 3D perovskite, the excited charge carriers can be trapped into the shallow traps in the orthorhombic phase, which can be de-trapped by increased thermal energy ${ }^{33}$. Even though the structural stabilization by the phase transition of $\mathrm{MAPbBr}_{3}$ can lead to the reduction in the density of the activated shallow trap states, deep level traps associated with ionic defects can still exist and cause nonradiative recombination. In contrast, $3 \mathrm{D} / 2 \mathrm{D}$ hybrid perovskite can have more efficient radiative recombination because the incorporation of $2 \mathrm{D}$ perovskite can lead to effective passivation by suppressing the formation and migration of the ionic defects thereby minimizing a loss of charge carrier from quenching by the deep traps.

To verify the reduced density of trap states in $3 \mathrm{D} / 2 \mathrm{D}$ hybrid perovskite compared to $3 \mathrm{D}$ perovskite, we designed and characterized hole-only devices (HODs) with a structure of ITO/Buf-HIL $(50 \mathrm{~nm}) /$ Perovskite $(400 \mathrm{~nm}) / \mathrm{MoO}_{3}(10 \mathrm{~nm}) / \mathrm{Au}$ $(100 \mathrm{~nm})$ (Fig. $5 \mathrm{f})$. The $I-V$ behaviour of the devices had three regimes defined by slope $k$ : an Ohmic regime $(k=1)$, a trap-filled limited (TFL) regime $(k>3)$ and a trap-free space charge limited current (SCLC) regime $(k=2)$ as the bias increased ${ }^{37-39}$. Here, trap density $n_{\mathrm{t}}$ is linearly proportional to trap-filled limit voltage $V_{\mathrm{TFL}}$ at which a transition of $I-V$ behaviour from Ohmic to TFL occurs:

$$
V_{\mathrm{TFL}}=n_{\mathrm{t}} \frac{e L^{2}}{2 \varepsilon \varepsilon_{0}}
$$

where $e$ is the electron charge, $L$ is the thickness of the perovskites, $\varepsilon$ is the relative dielectric constant (25.5 for $\mathrm{MAPbBr}_{3}$ ), and $\varepsilon_{0}$ is the vacuum permittivity ${ }^{40}$. In the $I-V$ curves, $V_{\text {TFL }}$ was lower in HODs based on $3 \mathrm{D} / 2 \mathrm{D}$ hybrid perovskite $(0.436 \mathrm{~V})$ than in HODs based on pristine $3 \mathrm{D}$ perovskite $(0.609 \mathrm{~V})$. Thus, we obtained $n_{\mathrm{t}}=9.49 \times 10^{15} \mathrm{~cm}^{-3}$ for $3 \mathrm{D} / 2 \mathrm{D}$ hybrid perovskite and $n_{\mathrm{t}}=1.33 \times 10^{16} \mathrm{~cm}^{-3}$ for $3 \mathrm{D}$ perovskite. This result supports that the formation of $2 \mathrm{D}$ perovskite can passivate the trap states of $3 \mathrm{D}$ perovskite leading to efficient radiative recombination. We also calculated the hole mobility $\mu_{\mathrm{h}}$ of each device by fitting the curves in the regime of trap-free SCLC based on the Mott-Gurney law:

$$
J=\frac{9 \varepsilon \varepsilon_{0} \mu_{\mathrm{h}} V^{2}}{8 L^{3}}
$$

where $V$ is applied voltage. The hole mobility of two devices had similar value $\mu_{\mathrm{h}}: 0.042 \mathrm{~cm}^{2} \mathrm{~V}^{-1} \mathrm{~s}^{-1}$ in the HOD with $3 \mathrm{D} / 2 \mathrm{D}$ hybrid perovskite, and $0.049 \mathrm{~cm}^{2} \mathrm{~V}^{-1} \mathrm{~s}^{-1}$ in the HOD device with $3 \mathrm{D}$ perovskite. This result indicates that the formation of $2 \mathrm{D}$ perovskite does not degrade hole transport in the perovskite.

EL characteristics of PeLEDs and ion migration. We used the developed perovskite films as an emitter in PeLEDs. The device structure was FTO/Buf-HIL $(50 \mathrm{~nm}) /$ perovskite $(400 \mathrm{~nm}) / 2,2^{\prime}, 2^{\prime}$ '-(1,3,5-benzinetriyl)-tris(1-phenyl-1-H-benzimidazole) (TPBi) $(50 \mathrm{~nm}) / \mathrm{LiF}(1 \mathrm{~nm}) / \mathrm{Al}(100 \mathrm{~nm})$ (Fig. 6a). All LED characteristics were significantly improved in the device with the $3 \mathrm{D} / 2 \mathrm{D}$ hybrid perovskite emitter compared to those of the device with the $3 \mathrm{D}$ perovskite emitter. The 3D/2D PeLED had maximum current efficiency $\mathrm{CE}_{\max }=20.55 \mathrm{~cd} \mathrm{~A}^{-1}$, and external quantum efficiency $\mathrm{EQE}_{\max }=4.23 \%$, which are both higher than in the 3D PeLED $\left(\mathrm{CE}_{\max }=13.23 \mathrm{~cd} \mathrm{~A}^{-1}\right.$ and $\left.\mathrm{EQE}_{\max }=2.50 \%\right)$ (Fig. 6b-e). Both devices had the same normalized EL emission spectra, which is consistent with the result of PL spectra (Fig. 6f). Devices were optimized by adjusting the amount of BnA (Supplementary Fig. 13 and Supplementary Table 5). We attribute the improvement in EL characteristics of 3D/2D hybrid PeLED to the efficient exciton confinement and the reduced defect-assisted recombination that result from the passivation of trap sites by the formation of $2 \mathrm{D}$ perovskite ${ }^{31}$. The effective suppression of ion migration by the $3 \mathrm{D} / 2 \mathrm{D}$ hybrid perovskite structure can also contribute to the EL improvement because charge carriers can result in emission of light instead of going through non-radiative recombination by the ionic space charges that are distributed at the vicinity of perovskite interfaces ${ }^{41}$. In contrast, the use of ANI-added perovskite film degraded the LED characteristics due to significant nonradiative recombination by the electron-deficient ANI as well as by the ionic space charges at the interfaces with the perovskiteemitting layer (Supplementary Fig. 14).

To verify that there is a different behaviour regarding ion migration between 3D/2D hybrid and 3D PeLEDs, we observed a time-dependent change in current under constant voltage (CV), and time-dependent change in voltage under constant current (CC). Under CV of $3.5 \mathrm{~V}$, the $3 \mathrm{D} / 2 \mathrm{D}$ hybrid PeLED maintained relatively stable current during measurement (Fig. 7a); this result is a consequence of suppressed ion migration, so the local electric field at the interfaces remains mild. In contrast, the 3D PeLED showed severe overshoot of current during the initial stage of the measurement; we attribute this phenomenon to rapidly facilitated charge injection that occurs because the accumulated ions at the interfaces cause narrowing of the depletion region width or lowering of the injection barrier ${ }^{42}$.

We also examined a voltage change of PeLEDs by varying the applied CC from 0.16 to $2.22 \mathrm{~mA} \mathrm{~cm}^{-2}$ step-wise by repeating switching on and off (Fig. 7b, c). The 3D/2D hybrid PeLED showed stable plateaus of voltage; this phenomenon proves that the device does not suffer severely from ion migration and accumulation. In contrast, the $3 \mathrm{D}$ PeLED showed a voltage undershoot at the beginning of each step, then recovery after the saturation threshold of ion migration. The undershoot of voltage can be explained by the facilitated charge injection, which instantly decreases the built-in potential of the device. The time to reach the saturation threshold of ion migration decreased from 25 to $5 \mathrm{~s}$ as CC was increased from 0.16 to $1.11 \mathrm{~mA} \mathrm{~cm}^{-2}$, but the further increase in CC did not cause undershoot in voltage; this result indicates that the migration can be accelerated by increasing the applied current. Magnification (Fig. 7c) of a part of the steps with the CC of $1.11 \mathrm{~mA} \mathrm{~cm}^{-2}$ clearly shows the difference in the stabilities of the devices and peculiar behaviour of the 3D PeLED.

To understand the behaviour of ion migration, we investigated the frequency-dependent capacitance of both types of PeLED in darkness (Fig. 7d). Both devices showed similar capacitance curves in the frequency range $100 \mathrm{~Hz} \leq f \leq 1 \mathrm{MHz}$, in which capacitance is a result of geometric parameters and dipolar polarization, but 3D PeLED showed increasing capacitance at $f<$ $100 \mathrm{~Hz}$. This additional capacitance may originate from mobile ions that accumulate at contact interfaces because of the severe ion migration 43,44 . The $3 \mathrm{D} / 2 \mathrm{D}$ hybrid PeLED did not show the additional capacitance; this result indicates that ion migration was effectively suppressed.

Here, we propose possible pathways of ion migration in $3 \mathrm{D}$ and $3 \mathrm{D} / 2 \mathrm{D}$ hybrid perovskite and a mechanism of ion migration suppression in the latter (Fig. 7e). The diffusion barrier energy within the $3 \mathrm{D}$ bulk region (Fig. 7e, red) can be considered similar in both systems because they have similar formation energy of ion defects in the $3 \mathrm{D}$ bulk. However, the barrier energy for ion diffusion becomes highly different when the migrating ions reach 
a
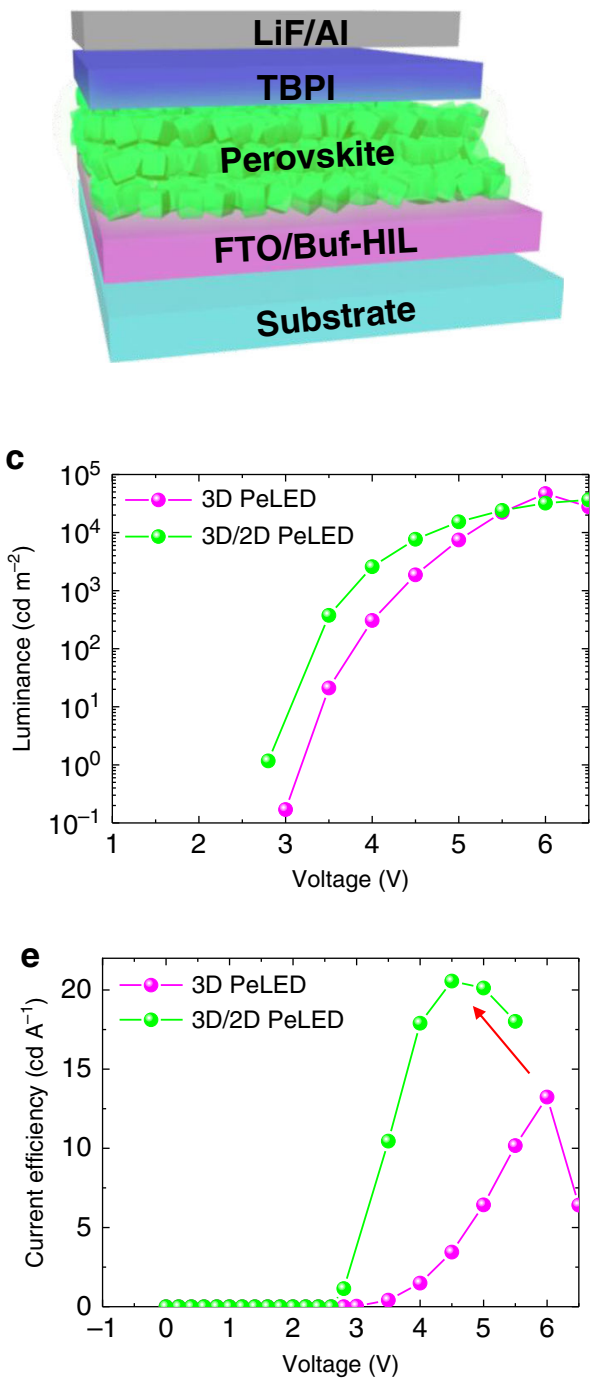
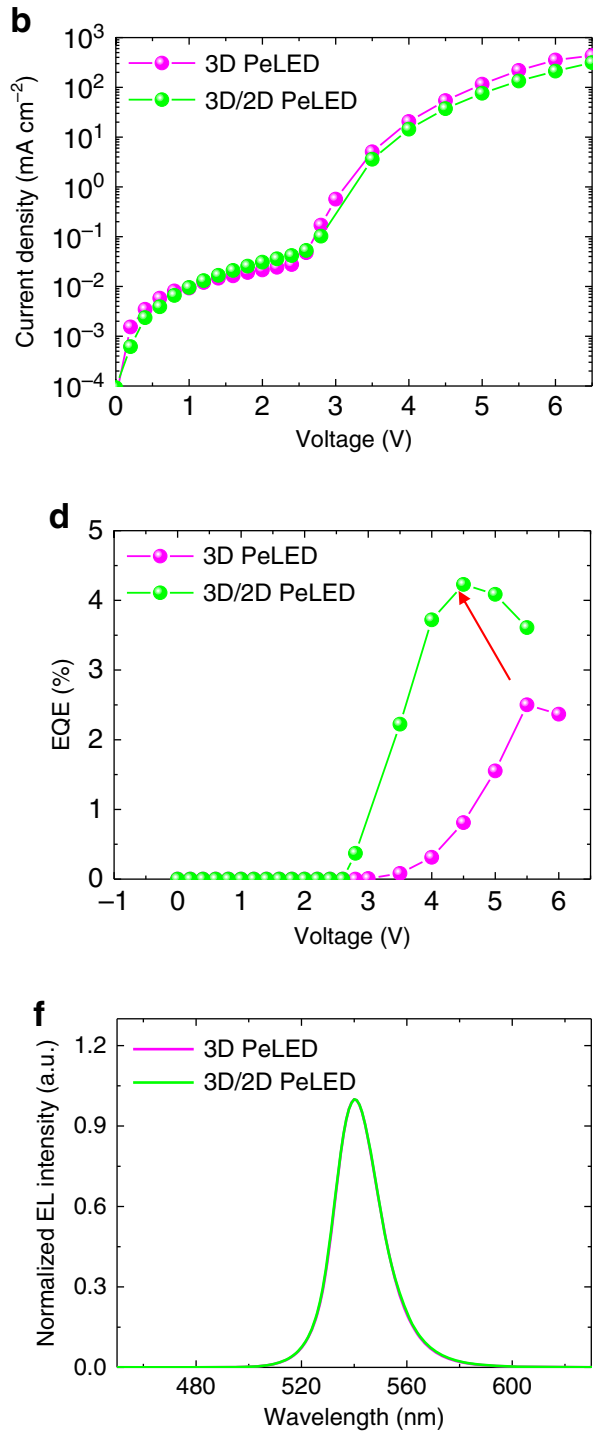

Fig. 6 EL characteristics of PeLEDs. a Device structure of PeLEDs. b Current density of PeLEDs as a function of applied voltage. $\mathbf{c}$ Luminance of PeLEDs as a function of applied voltage. d External quantum efficiency (EQE) of PeLED as a function of applied voltage. e Current efficiency (CE) of PeLEDs as a function of applied voltage. $\mathbf{f}$ Normalized EL spectra of PeLEDs.

the GB, where ion migration most easily occurs (Fig. 7e, green). In $3 \mathrm{D}$ perovskite, the ions can reach the $\mathrm{GB}$ without being required to diffuse across the $2 \mathrm{D}$ perovskite barrier layer. In $3 \mathrm{D} /$ 2D hybrid perovskite, on the contrary, the ions should overcome the higher barrier energy to get over the organic capping in the $2 \mathrm{D}$ perovskite region (Fig. 7e, purple). We summarized the possible pathways of ion migration in the perovskites and presented a qualitative comparison of the energy barrier for ion migration between $3 \mathrm{D}$ perovskite and $3 \mathrm{D} / 2 \mathrm{D}$ hybrid perovskite (Supplementary Table 6). Also, the 3D/2D hybrid perovskite can effectively suppress the ion migration due to the morphological advantage observed by scanning electron microscopy (SEM) images (Fig. 3d). As the ion migration mostly occurs on GBs, mobile ions have to migrate much longer pathway in the granular-like 3D/2D hybrid perovskite than in the columnar-like $3 \mathrm{D}$ perovskite (Supplementary Fig. 15). Furthermore, the 3D/2D hybrid perovskite has a much greater number of boundary nodes where migrating ions along the direction of the external electric field can be blocked and lose their kinetic energy leading to the termination of their migration. In order to scrutinize the structure of the $3 \mathrm{D} / 2 \mathrm{D}$ hybrid perovskites in grain scale, we used highangle annular dark-field scanning transmission electron microscopy (HAADF-STEM) and clearly observed the 2D perovskite layer with $n=1$ (Supplementary Fig. 16).

$\mathrm{Ab}$ initio $\mathrm{MD}$ simulations predicted that ion migration in perovskites can be greatly assisted by $\mathrm{MA}^{+}$reorientation in response to motion of the halide ion ${ }^{36}$. For example, the rotation of $\mathrm{MA}^{+}$can lead to the displacement of a halide ion along the direction of the $\mathrm{C}-\mathrm{N}$ axis of $\mathrm{MA}^{+}$based on their attractive Coulomb force. Thus, the incorporation of less orientationally mobile cations with a lower rotational degree of freedom than $\mathrm{MA}^{+}$can reduce ion migration in perovskites. In this regard, $\mathrm{BnA}^{+}$can have remarkably reduced reorientation rate by themselves based on their $\pi-\pi$ interaction and steric repulsion as a result of neighbouring benzene rings; this slowing of $\mathrm{BnA}^{+}$ reorientation in the perovskite lattice can effectively impede ions migration along GBs compared to $\mathrm{MA}^{+45}$.

Combining the simulation results with the transition-state theory of rate processes, we can predict the retardation effect of $\mathrm{BnA}^{+}$relative to $\mathrm{MA}^{+}$on the ion migration rate ${ }^{45}$. We 
a

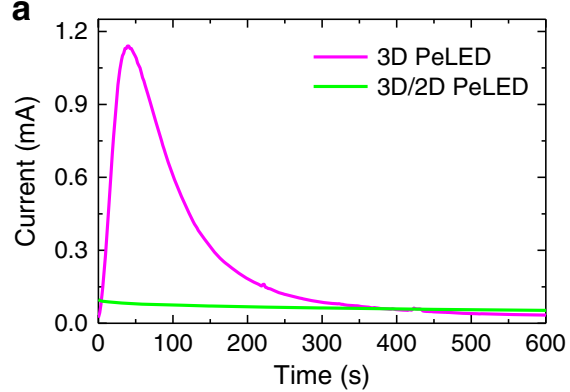

c

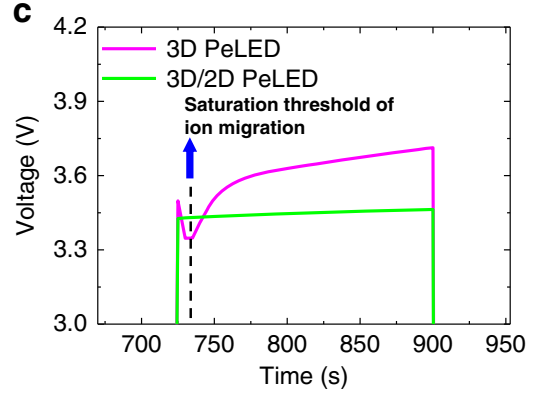

b
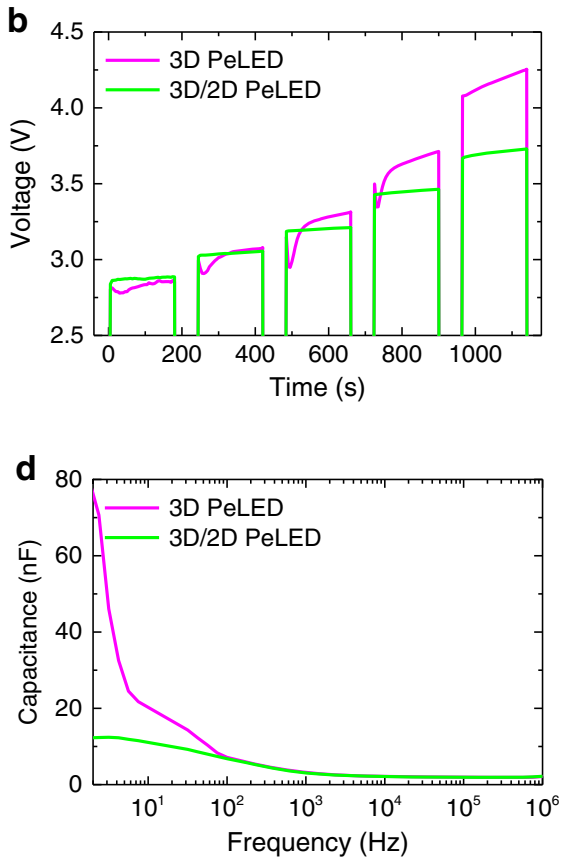

e

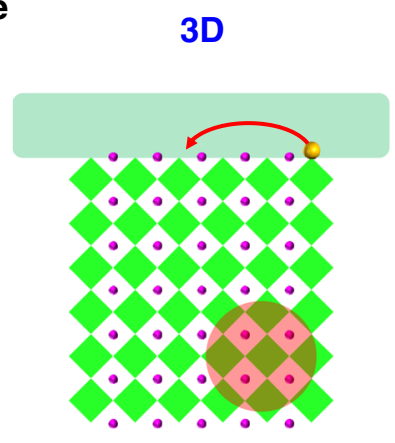

3D/2D Hybrid

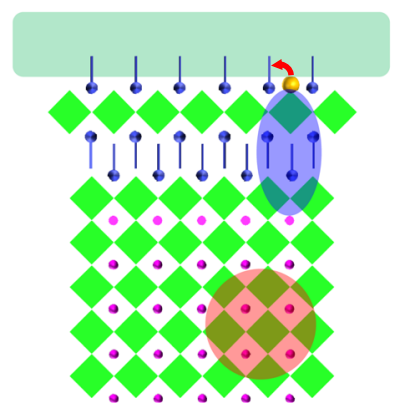

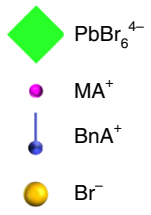

Available pathways of ion migration

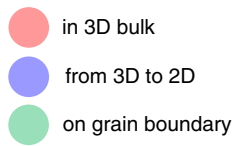

Fig. 7 Influence of ion migration in perovskites on PeLEDs. a Current behaviour of PeLEDs as a function of time under constant voltage of $3.5 \mathrm{~V}$. b Voltage behaviour of PeLEDs under increasing step constant current of $0.16,0.22,0.44,1.11,2.22 \mathrm{~mA} \mathrm{~cm}^{-2}$ from the left column to the right in a sequence. c Magnified version of one step showing the discrepancy in the voltage behaviour between 3D PeLED and 3D/2D hybrid PeLED. d Capacitance-frequency curve of PeLEDs. e Schematic of possible ion migration pathways in 3D and 3D/2D hybrid perovskites and efficient restriction of ion migration owing to the $\mathrm{BnA}$ which has retardation effect of reorientation.

examined this retardation effect more concretely by considering atomic-bond breaking associated with the organic-cation reorientation. In $\mathrm{MAPbX}_{3}$, the two dominant hydrogen bonds should be broken to allow $180^{\circ}$ reorientation of an $\mathrm{MA}^{+}$adjacent to a migrating defect ion ${ }^{46}$. We defined the energy of the dominant hydrogen bond between a proton in $\mathrm{NH}_{3}$ and halide ion in the $\mathrm{PbX}_{6}$ octahedron unit as $E_{\mathrm{HB}}$. Previous ab initio calculations based on quantum mechanics of atoms in molecule (QTAIM) obtained $E_{\mathrm{HB}} \approx 200 \mathrm{meV} / \mathrm{MA}^{+}$for $\mathrm{MAPbI}_{3}{ }^{46,47}$. In $\mathrm{BnA}^{+}$, the breaking of the $\pi-\pi$ interaction themselves should be considered, in addition to the rupturing of this hydrogen bonding. According to ab initio calculations based on the density-functional tight-binding (DFTB) method, the energy $E_{\pi \pi}$ of the $\pi-\pi$ stacking interaction between two neighbouring benzene rings is $-4.38 \mathrm{kcal} \mathrm{mol}^{-148}$. For simplicity, we assume that $E_{\mathrm{HB}}$ of $\mathrm{BnA}^{+}$is approximately equal to that of $\mathrm{MA}^{+}$. This assumption does not alter our qualitative argument on the retardation effect caused by the substitution of $\mathrm{BnA}^{+}$for $\mathrm{MA}^{+}$. We define the rate (frequency per unit time) of the reorientation of $\mathrm{BnA}^{+}$and $\mathrm{MA}^{+}$as $v_{\mathrm{BnA}}$ and $v_{\mathrm{MA}}$, respectively, and the retardation coefficient $\left(R_{\mathrm{c}}\right)$ as the ratio of these two frequencies.
According to the transition-state theory,

$$
\nu_{\mathrm{BnA}}=\frac{R T}{N_{\mathrm{o}} h} \mathrm{e}^{-\left(E_{\mathrm{HB}}+2 E_{\pi \pi}\right) /(R T)},
$$

where $N_{\mathrm{o}}$ is Avogadro's number, $h$ is Planck's constant, and $R$ denotes the gas constant $\left(=1.987 \mathrm{cal} \mathrm{mol}^{-1} \mathrm{~K}^{-1}\right)^{45}$; Here, a factor of ' 2 ' is introduced in the exponent by considering that two $\pi-\pi$ stackings must be broken for the reorientation of each $\mathrm{BnA}^{+}$in the $3 \mathrm{D} / 2 \mathrm{D}$ hybrid structure. Then the retardation coefficient can be written as $R_{\mathrm{c}} \equiv \frac{\nu_{\mathrm{BAA}}}{\nu_{\mathrm{MA}}} \approx e^{-2 E_{\pi \pi} /(R T)}=4.23 \times 10^{-7}$ at $300 \mathrm{~K}$. This is only an order-of-magnitude estimate, but it suggests strongly that the substitution of $\mathrm{BnA}^{+}$for $\mathrm{MA}^{+}$remarkably reduces the reorientation rate and, thereby significantly suppresses ion migration.

Stability of PeLEDs and ion migration. The 3D/2D hybrid PeLEDs also showed the excellent $I-V-L$ reliability under the repetition of sweeping voltage from 0 to $5 \mathrm{~V}$ and remarkably maintained its current density and luminance in spite of the repeating voltage sweep over 20 times (Fig. $8 \mathrm{a}-\mathrm{d}$ ). This 

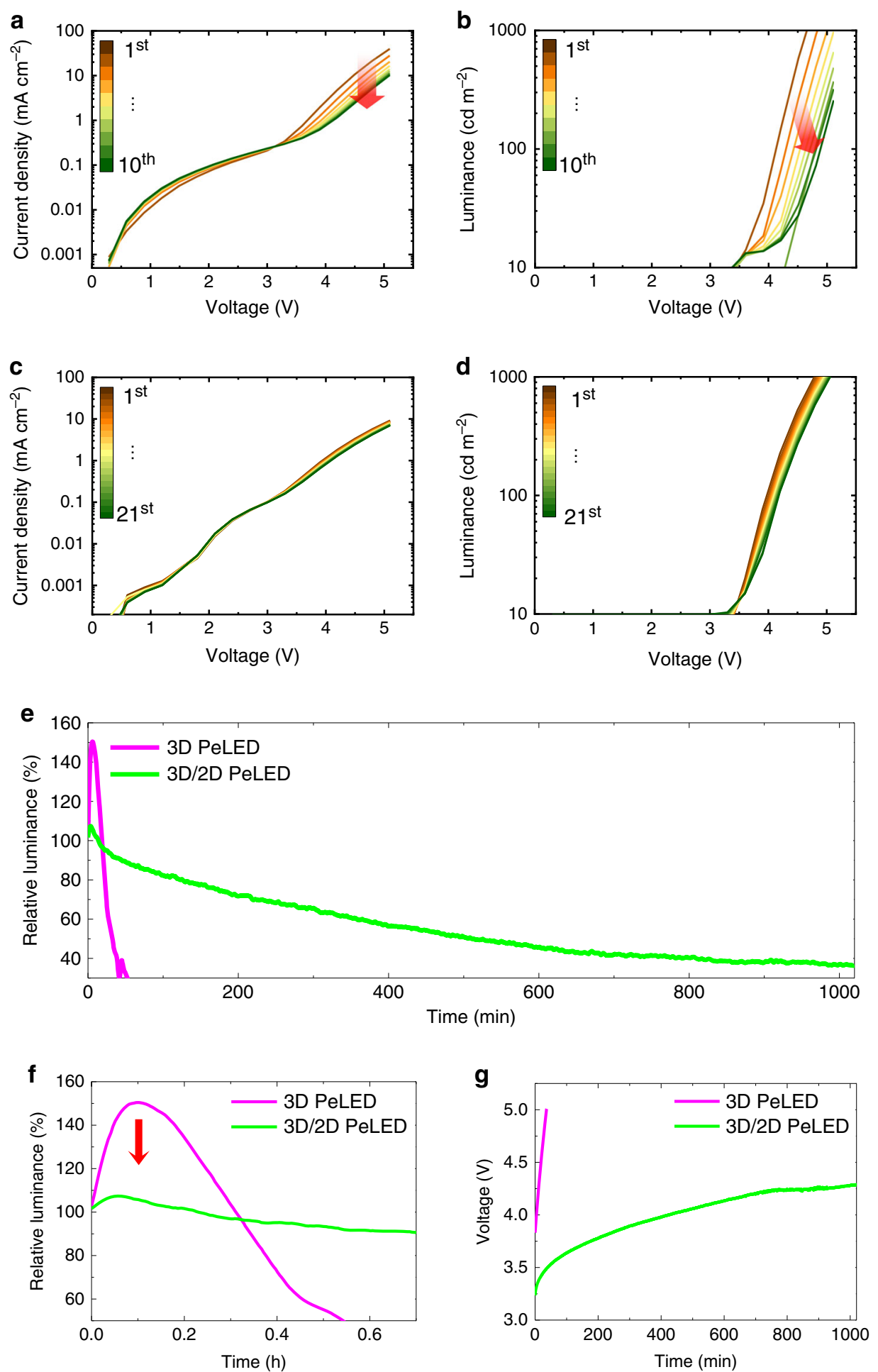

Fig. 8 Reliability and long-term stability of PeLEDs. a $J-V$ and $\mathbf{b} L-V$ curves of 3D PeLEDs with a repetition of voltage sweep and $\mathbf{c}$, $\mathbf{d}$ those of 3D/2D hybrid PeLED. e Relative luminance of 3D/2D hybrid PeLED (green) and of 3D PeLED (red) over time. f Magnified version of relative luminance of PeLEDs showing an extremely reduced overshoot of luminance. $\mathbf{g}$ Voltage curves of 3D/2D hybrid PeLED (green) and 3D PeLED (red) over time under constant current to make PeLEDs have an emission of $100 \mathrm{~cd} \mathrm{~m}^{-2}$.

characteristic is a result of the suppressed ion migration. In contrast, the 3D PeLED showed degradation in current density and luminance in $<10$ such voltage sweeps. The $3 \mathrm{D} / 2 \mathrm{D}$ hybrid PeLED also showed much less $J-V$ hysteresis than the 3D PeLED under continuous forward and backward scans (Supplementary Fig. 17). This excellence of the stability demonstrates that the 3D/ 2D hybrid PeLED has much better operational stability than the
3D PeLED. The measurement was performed under CC condition for each device to elicit luminance of $100 \mathrm{~cd} \mathrm{~m}^{2}$ (Fig. 8e). The luminance of the $3 \mathrm{D}$ PeLED overshot to $150.4 \%$ of the initial luminance, then dropped to $40 \%$ of the luminance after time $T_{40}=38 \mathrm{~min}$. As ions migrate towards and accumulate at each end of the perovskite layer, the charge injection from the electrodes tends to be facilitated because of the strong local 
electric field at the interfaces, and this facilitation results in the initial luminance overshoot. In contrast, the $3 \mathrm{D} / 2 \mathrm{D}$ hybrid PeLED had a dramatically increased operating lifetime as $T_{40}=$ $810 \mathrm{~min}$, which is more than 21 times longer than $T_{40}$ of $3 \mathrm{D}$ PeLED. Moreover, the 3D/2D hybrid PeLED showed extremely reduced luminance overshoot as only $7.4 \%$ increase from the initial luminance, because the ion migration was effectively suppressed (Fig. 8f). We also investigated the operational stability of the PeLED using the ANI-added $\mathrm{MAPbBr}_{3}$, which showed strong luminance overshoot as $146.7 \%$ compared to the initial luminance, implying that the addition of ANI cannot effectively suppress the ion migration (Supplementary Fig. 18). We summarized the luminance overshoot of PeLEDs from reported studies and compared their overshoot ratios (Supplementary Table 7 and Supplementary Fig. 19).

The applied voltage in the devices also showed a large difference as a function of time (Fig. 8g). The steeper voltage increase in the 3D PeLED indicates the inferior stability of the device compared to the $3 \mathrm{D} / 2 \mathrm{D}$ hybrid PeLED. Also, accumulated ions at the interface can cause chemical corrosion of electrodes and thereby accelerate the degradation of the device ${ }^{49}$. The $3 \mathrm{D} / 2 \mathrm{D}$ hybrid PeLED does not suffer from those problems because of the effectively suppressed ion migration resulting in the much longer operating lifetime than the $3 \mathrm{D}$ perovskite PeLED.

\section{Discussion}

A perovskite light-emitter of proton-transfer-induced 3D/2D hybrid structure composed of dominant $3 \mathrm{D} \mathrm{MAPbBr}_{3}$ and a small amount of $2 \mathrm{D} \mathrm{BnA}{ }_{2} \mathrm{PbBr}_{4}$ has been developed by using a neutral amine reagent $\mathrm{BnA}$ instead of ammonium halide salt. A proton-transfer reaction between $\mathrm{MA}^{+}$and $\mathrm{BnA}$ occurs in the precursor state, and facilitates participation by $\mathrm{BnA}^{+}$in $2 \mathrm{D}$ perovskite crystallization. A $2 \mathrm{D}$ phase forms without destroying the $3 \mathrm{D}$ perovskite phase in the film. The incorporation of $2 \mathrm{D}$ perovskite into 3D perovskite restricts the formation of ionic defects because $\mathrm{BnA}^{+}$has a lower rotational degree of freedom than $\mathrm{MA}^{+}$. Therefore, $3 \mathrm{D} / 2 \mathrm{D}$ hybrid perovskite leads to efficient radiative recombination by deactivating even deep-level trap states. Also, the $3 \mathrm{D} / 2 \mathrm{D}$ hybrid structure can suppress ion migration owing to the retardation effect in the reorientation of $\mathrm{BnA}^{+}$in the lattice based on $\pi-\pi$ interaction of their aromatic rings by considering possible pathways of ion migration. The PeLEDs with the $3 \mathrm{D} / 2 \mathrm{D}$ hybrid perovskite emitter show an extremely reduced initial luminance overshoot ratio as 7.4\%, 21 times longer operational stability without noticeable overshoot of luminance, and significantly improved luminous efficiency. The material insights on 'proton-induced 3D/2D hybrid perovskites' we propose can help pave the way for the future practical applications of perovskite electronics (i.e. solar cells, LEDs, and memories) with a long operating lifetime.

\section{Methods}

Materials. TPBi was purchased from OSM and MABr was purchased from Greatcell Solar. $\mathrm{PbBr}_{2}$ (99.999\%), ANI (ACS Reagent, 99.5\%), BnA (ReagentPlus, $99 \%), \operatorname{LiF}(99.99 \%$, trace metal basis), DMSO (99.8\%, anhydrous) were purchased from Sigma-Aldrich. All chemicals were used as received.

Preparation of perovskite precursor solution. $\mathrm{MAPbBr}_{3}$ precursor solutions (35.0 wt\%) were prepared by dissolving $\mathrm{MABr}$ and $\mathrm{PbBr}_{2}$ in DMSO with a molar ratio of 1.06:1 ( $\left.\mathrm{MABr}: \mathrm{PbBr}_{2}\right)$, and then stirred overnight. Before spin-coating the solution, the neutral amine additive (ANI or BnA) was added into the $\mathrm{MAPbBr}_{3}$ precursor solution.

Device fabrication. For PeLEDs, the Buf-HIL layer was spin-coated on a cleaned FTO-coated glass at $3000 \mathrm{rpm}$ for $90 \mathrm{~s}$, and samples were annealed on a hot plate for $30 \mathrm{~min}$ at $150^{\circ} \mathrm{C}$ in air. Buf-HIL solution was composed of PEDOT:PSS

(Clevios P VP AI4083) and tetrafluoroethylene-perfluoro-3,6-dioxa-4-methyl-7-

octene-sulfonic acid copolymer (PFI) (Sigma-Aldrich) (1:1 wt:wt). On the prepared substrate, the prepared perovskite precursor solution was spin-coated at $3000 \mathrm{rpm}$ followed by the additive-based nanocrystal-pinning process to induce immediate crystallization of perovskite by using TPBi solution in chloroform ${ }^{3}$. The deposited perovskite film was annealed on a hot plate at $90^{\circ} \mathrm{C}$ for $10 \mathrm{~min}$. Subsequently, 50 $\mathrm{nm}$ of TPBI, $1 \mathrm{~nm}$ of $\mathrm{LiF}$, and $100 \mathrm{~nm}$ of $\mathrm{Al}$ were thermally evaporated in sequence in a high-vacuum chamber to complete the device. For HODs, $30 \mathrm{~nm}$ of molybdenum oxide $\left(\mathrm{MoO}_{3}\right)$ and $50 \mathrm{~nm}$ of Au were thermally deposited on top of $\mathrm{MAPbBr}$ to make a structure of glass/FTO/Buf-HIL/MAPbBr $3 / \mathrm{MoO}_{3} / \mathrm{Au}$ in the high-vacuum chamber. The devices were encapsulated in $\mathrm{N}_{2}$ atmosphere and the pixel area of a device was $4.8 \mathrm{~mm}^{2}$.

${ }^{1}$ H NMR measurement. The ${ }^{1} \mathrm{H}$ NMR spectra were obtained using a highresolution NMR spectrometer (Bruker Advance $600 \mathrm{MHz}$ ). For the measurement, the perovskite precursor solution was prepared as mentioned above, except that the precursors were dissolved in DMSO- $d_{6}(1 \mathrm{~mL})$. For additive-mixed solutions, different amounts of BnA or ANI were added to the precursor solution as the experimental sample. The samples were prepared at room temperature in $\mathrm{N}_{2}$ atmosphere, and their characteristics were measured at room temperature.

1H MAS NMR spectroscopy. ${ }^{1} \mathrm{H}$ MAS NMR spectra of the samples were collected at room temperature on a Bruker NMR system (14.1 T) at Larmor frequency of $600.41 \mathrm{MHz}$ with a $1.9-\mathrm{mm}$ triple-resonance Bruker NMR probe using a spin-echo pulse sequence at a spinning speed of $40 \mathrm{kHz}$. Fast sample spinning speed of $40 \mathrm{kHz}$ yielded high-resolution ${ }^{1} \mathrm{H}$ MAS NMR spectra. The spin-echo pulse sequence $(\pi / 2-\mathrm{t}-\pi-\mathrm{t})$ was used to suppress ${ }^{1} \mathrm{H}$ background signals from the probe ${ }^{50,51}$. The $\pi / 2$ pulse length of $2 \mu$ s was applied for the samples and a recycle delay time of $10 \mathrm{~s}$ was used. Approximately $0.5-6 \mathrm{mg}$ of perovskite powder after being scraped off the film was used. To improve the signal-to-noise ratio, around 16-256 scans were averaged in the ${ }^{1} \mathrm{H}$ MAS NMR spectra. The ${ }^{1} \mathrm{H}$ NMR spectra were referenced externally using tetramethylsilane (TMS) solution.

Characterizations of perovskite films. The absorbance of the perovskite film was measured using UV-vis absorption spectrophotometer (Cary-5000). Steady-state PL of the film was measured using a spectrofluorometer (JASCO FP6500). Xenon arc lamp with continuous output power of $150 \mathrm{~W}$ was used as an excitation source, and the excitation wavelength was $365 \mathrm{~nm}$. The light was incident from the glass side. XRD analysis was carried out using X-ray diffractometer (PANalytical) with $\mathrm{Cu}$ ka radiation at a scan rate of $4 \% \mathrm{~min}$. GIXD was performed on the perovskite film at the 6D and 9A beamlines at the Pohang Accelerator Laboratory, Korea. Top-view and cross-sectional SEM images of the perovskite films were obtained using a field-emission SEM (MERLIN compact, ZEISS) at the Research Institute of Advanced Materials in Seoul National University. TCSPC measurement was conducted by using TCSPC module (FluoTime 300, PicoQuant). The instrument response function (IRF) was ignored because the PL lifetime curves were much longer than the temporal width of the IRF. The excitation power density was around $25 \mathrm{~mW} \mathrm{~cm}^{-2}$, and the excitation wavelength was fixed at $405 \mathrm{~nm}$. The excitation power density was determined by using a laser power meter (Thorlabs) for the excitation-dependent PL lifetime measurement. For the temperaturedependent PL measurement, the sample was mounted in a cryostat under vacuum condition. The target temperature was equilibrated by over $3 \mathrm{~min}$ stabilization before measuring a steady-state PL spectrum. UPS of the perovskite films was measured using a photoelectron spectrometer (Kratos Inc., AXIS-Ultra DLD) with $\mathrm{He}$ I radiation $(21.2 \mathrm{eV})$ in collaboration with Korea Basic Science Institute (KBSI). PLQE of perovskite thin films on quartz substrate was measured by using the integrating sphere method with a continuous-wave $405 \mathrm{~nm}$ laser diode $\left(2.5 \mathrm{~mW} \mathrm{~cm}^{-2}\right)^{3}$

Transmission electron microscopy (TEM) analysis. HAADF-STEM and energydispersive X-ray spectrum (EDXS) imaging were performed on an aberrationcorrected Titan-Themis 60-300 (Thermo Scientific) at an acceleration voltage of $200 \mathrm{kV}$ with probe currents of approximately 100 and $500 \mathrm{pA}$, respectively. This microscope is equipped with a high brightness Schottky X-FEG gun, a Super-X EDX system comprising four silicon drift detectors, and Velox acquisition software. The specimen for STEM analysis was prepared on a carbon support grid by dripping solution which was a dispersion of perovskite powder in chloroform after being scraped off the film.

Characterization of PeLEDs. The current-voltage-luminance characteristics of PeLEDs were measured using a source-measurement unit (Keithley 236), a spectroradiometer (Minolta CS-2000), and a control computer. The operational lifetime of the PeLEDs was measured using a lifetime measurement system (M6000, McScience) under a CC condition that yielded luminance of $100 \mathrm{~cd} \mathrm{~m}^{-2}$. Capacitance-voltage, capacitance-frequency, and time-dependent $J-V$ characteristics were measured using a Solartron 1260 impedance/gain-phase analyzer with a Solartron 1287 potentiostat.

Charge-density calculation details. Charge-density calculations were conducted using a plane-wave pseudopotential approach within DFT as implemented in the 
Viena Ab Initio Simulation Package ${ }^{52}$. The electron-core interactions were described with the projected-augmented wave pseudopotentials and the exchange and correlation functional were treated using the Perdew-Burke-Ernzerhof parametrization of GGA for structural relaxations ${ }^{53,54}$. The molecule was placed in the middle of a size-fixed cubic cell with $a=b=c=30 \AA$ to optimize the atomic configuration. The electronic energy was minimized with a tolerance of $10^{-6} \mathrm{eV}$, and ionic relaxation was performed with a force tolerance of $0.01 \mathrm{eV} \AA^{-1}$ on each ion. The kinetic energy cutoff was set as $520 \mathrm{eV}$, and a Gamma point was used for the Brillouin zone integration. The charge density along $b$-axis was integrated from the CHGCAR by $\rho(y)=\int \rho(x, y, z) \mathrm{d} x \mathrm{~d} z$.

\section{Data availability}

The data that support the findings of this study are available from the corresponding author upon reasonable request.

Received: 27 August 2019; Accepted: 2 June 2020;

Published online: 06 July 2020

\section{References}

1. Tan, Z. et al. Bright light-emitting diodes based on organometal halide perovskite. Nat. Nanotechnol. 9, 687-692 (2014).

2. Kim, Y.-H. et al. Multicolored organic/inorganic hybrid perovskite lightemitting diodes. Adv. Mater. 27, 1248-1254 (2015).

3. Cho, H. et al. Overcoming the electroluminescence efficiency limitations of perovskite light-emitting diodes. Science 350, 1222-1225 (2015).

4. Lin, K. et al. Perovskite light-emitting diodes with external quantum efficiency exceeding 20 per cent. Nature 562, 245-248 (2018).

5. Zhao, B. et al. High-efficiency perovskite-polymer bulk heterostructure lightemitting diodes. Nat. Photonics 12, 783-789 (2018).

6. Cao, Y. et al. Perovskite light-emitting diodes based on spontaneously formed submicrometre-scale structures. Nature 562, 249-253 (2018).

7. Zhao, L. et al. Electrical stress influences the efficiency of $\mathrm{CH}_{3} \mathrm{NH}_{3} \mathrm{PbI}_{3}$ perovskite light emitting devices. Adv. Mater. 29, 1605317 (2017).

8. Zhao, L. et al. In situ preparation of metal halide perovskite nanocrystal thin films for improved light-emitting devices. ACS Nano 11, 3957-3964 (2017).

9. Yuan, Y. \& Huang, J. Ion migration in organometal trihalide perovskite and its impact on photovoltaic efficiency and stability. Acc. Chem. Res. 49, 286-293 (2016).

10. Lin, Y. et al. Suppressed ion migration in low-dimensional perovskites. ACS Energy Lett. 2, 1571-1572 (2017).

11. Eames, C. et al. Ionic transport in hybrid lead iodide perovskite solar cells. Nat. Commun. 6, 7497 (2015).

12. Tress, W. et al. Understanding the rate-dependent $J-V$ hysteresis, slow time component, and aging in $\mathrm{CH} 3 \mathrm{NH} 3 \mathrm{PbI} 3$ perovskite solar cells: the role of a compensated electric field. Energy Environ. Sci. 8, 995-1004 (2015).

13. Yun, J. S. et al. Critical role of grain boundaries for ion migration in formamidinium and methylammonium lead halide perovskite solar cells. Adv. Energy Mater. 6, 1600330 (2016).

14. Tsai, H. et al. Stable light-emitting diodes using phase-pure ruddlesdenpopper layered perovskites. Adv. Mater. 30, 1704217 (2018).

15. Yantara, N. et al. Designing efficient energy funneling kinetics in Ruddlesden-Popper perovskites for high-performance light-emitting diodes. Adv. Mater. 30, 1800818 (2018).

16. Quan, L. N. et al. Tailoring the energy landscape in quasi-2D halide perovskites enables efficient green-light emission. Nano Lett. 17, 3701-3709 (2017).

17. Cheng, Z. \& Lin, J. Layered organic-inorganic hybrid perovskites: structure, optical properties, film preparation, patterning and templating engineering. CrystEngComm 12, 2646-2662 (2010).

18. Zhou, N. et al. The spacer cations interplay for efficient and stable layered 2D perovskite solar cells. Adv. Energy Mater. 10, 1901566 (2020)

19. Shang, Y., Li, G., Liu, W. \& Ning, Z. Quasi-2D inorganic $\mathrm{CsPbBr}_{3}$ perovskite for efficient and stable light-emitting diodes. Adv. Funct. Mater. 28, 1801193 (2018).

20. Duim, $H$. et al. Mechanism of surface passivation of methylammonium lead tribromide single crystals by benzylamine. Appl. Phys. Rev. 6, 031401 (2019).

21. Byun, J. et al. Efficient visible quasi-2D perovskite light-emitting diodes. $A d v$. Mater. 28, 7515-7520 (2016)

22. Xiao, Z. et al. Efficient perovskite light-emitting diodes featuring nanometresized crystallites. Nat. Photonics 11, 108-115 (2017).

23. Yang, X. et al. Efficient green light-emitting diodes based on quasi-twodimensional composition and phase engineered perovskite with surface passivation. Nat. Commun. 9, 570 (2018).
24. Yang, X. et al. Effects of organic cations on the structure and performance of quasi-two-dimensional perovskite-based light-emitting diodes. J. Phys. Chem. Lett. 10, 2892-2897 (2019).

25. Lee, H.-D. et al. Efficient Ruddlesden-Popper perovskite light-emitting diodes with randomly oriented nanocrystals. Adv. Funct. Mater. 29, 1901225 (2019).

26. Park, M.-H. H. et al. Unravelling additive-based nanocrystal pinning for high efficiency organic-inorganic halide perovskite light-emitting diodes. Nano Energy 42, 157-165 (2017).

27. Miyadera, T. et al. Crystallization dynamics of organolead halide perovskite by real-time X-ray diffraction. Nano Lett. 15, 5630-5634 (2015).

28. Koh, T. M. et al. Nanostructuring mixed-dimensional perovskites: a route toward tunable, efficient photovoltaics. Adv. Mater. 28, 3653-3661 (2016).

29. Yuan, M. et al. Perovskite energy funnels for efficient light-emitting diodes. Nat. Nanotechnol. 11, 872-877 (2016).

30. He, C. et al. Turn on fluorescence sensing of vapor phase electron donating amines via tetraphenylporphyrin or metallophenylporphrin doped polyfluorene. Chem. Commun. 46, 7536-7538 (2010).

31. Chen, Z. et al. Recombination dynamics study on nanostructured perovskite light-emitting devices. Adv. Mater. 30, 1801370 (2018).

32. Droseros, N. et al. Origin of the enhanced photoluminescence quantum yield in $\mathrm{MAPbBr}_{3}$ perovskite with reduced crystal size. ACS Energy Lett. 3, $1458-1466$ (2018).

33. $\mathrm{Fu}$, J. et al. Localized traps limited recombination in lead bromide perovskites. Adv. Energy Mater. 9, 1803119 (2019).

34. Shi, J. et al. Identification of high-temperature exciton states and their phasedependent trapping behaviour in lead halide perovskites. Energy Environ. Sci. 11, 1460-1469 (2018).

35. Meggiolaro, D. et al. Iodine chemistry determines the defect tolerance of leadhalide perovskites. Energy Environ. Sci. 11, 702-713 (2018).

36. Mosconi, E. \& De Angelis, F. Mobile ions in organohalide perovskites: interplay of electronic structure and dynamics. ACS Energy Lett. 1, 182-188 (2016).

37. Bube, R. H. Trap density determination by space-charge-limited currents. J. Appl. Phys. 33, 1733-1737 (1962).

38. Dong, Q. et al. Electron-hole diffusion lengths $>175 \mu \mathrm{m}$ in solution-grown CH3NH3PbI3 single crystals. Science 347, 967-970 (2015).

39. Shi, D. et al. Low trap-state density and long carrier diffusion in organolead trihalide perovskite single crystals. Science 347, 519-522 (2015).

40. Saidaminov, M. I. et al. High-quality bulk hybrid perovskite single crystals within minutes by inverse temperature crystallization. Nat. Commun. 6, 7586 (2015).

41. Kim, H. et al. Hybrid perovskite light emitting diodes under intense electrical excitation. Nat. Commun. 9, 4893 (2018).

42. Lim, K.-G., Choi, M.-R. \& Lee, T.-W. Improvement of both efficiency and stability in organic photovoltaics by using water-soluble anionic conjugated polyelectrolyte interlayer. Mater. Today Energy 5, 66-71 (2017).

43. Kim, H.-S. \& Park, N.-G. Parameters affecting $I-V$ hysteresis of $\mathrm{CH}_{3} \mathrm{NH}_{3} \mathrm{PbI}$ perovskite solar cells: effects of perovskite crystal size and mesoporous $\mathrm{TiO}_{2}$ layer. J. Phys. Chem. Lett. 5, 2927-2934 (2014).

44. Almora, O. et al. Capacitive dark currents, hysteresis, and electrode polarization in lead halide perovskite solar cells. J. Phys. Chem. Lett. 6 , 1645-1652 (2015).

45. Strehlow, H. Fundamentale Kinetik: basic chemical kinetics. Von H. Eyring, S. H. Lin and S. M. Lin. John Wiley \& Sons, New York - Chichester - Brisbane Toronto 1980. VU, 493 S., zahlr. Formeln, geb., £ 20,-. Nachr. Chem. Tech. Lab. 28, 754-755 (1980).

46. Lee, J. H., Lee, J.-H., Kong, E.-H. \& Jang, H. M. The nature of hydrogenbonding interaction in the prototypic hybrid halide perovskite, tetragonal $\mathrm{CH}_{3} \mathrm{NH}_{3} \mathrm{PbI}_{3}$. Sci. Rep. 6, 21687 (2016).

47. Bader, R. Atoms in MoleculesĐA Quantum Theory (Clarendon, 1990).

48. Zhou, R. Carbon nanotubes. In: Modeling of Nanotoxicity: Molecular Interactions of Nanomaterials with Bionanomachines (ed. Zhou, R.) 45-59 (Springer International Publishing, 2015).

49. Back, H. et al. Achieving long-term stable perovskite solar cells via ion neutralization. Energy Environ. Sci. 9, 1258-1263 (2016).

50. Kweon, J. J. et al. Evidence from $900 \mathrm{MHz}{ }^{1} \mathrm{H}$ MAS NMR of displacive behavior of the model order-disorder antiferroelectric $\mathrm{NH}_{4} \mathrm{H}_{2} \mathrm{AsO}_{4}$. J. Phys. Chem. C 119, 5013-5019 (2015).

51. Kweon, J. J., Fu, R., Steven, E., Lee, C. E. \& Dalal, N. S. High field MAS NMR and conductivity study of the superionic conductor $\mathrm{LiH}_{2} \mathrm{PO}_{4}$ : critical role of physisorbed water in its protonic conductivity. J. Phys. Chem. C 118, 13387-13393 (2014).

52. Kresse, G. \& Furthmüller, J. Efficient iterative schemes for ab initio totalenergy calculations using a plane-wave basis set. Phys. Rev. B 54, 11169-11186 (1996).

53. Blochi, P. E. Projector augmented-wave method. Phys. Rev. B 50, 17953-17979 (1994) 
54. Kresse, G. \& Joubert, D. From ultrasoft pseudopotentials to the projector augmented-wave method. Phys. Rev. B 59, 11-19 (1999).

\section{Acknowledgements}

This work was supported by the National Research Foundation of Korea (NRF) grant funded by the Korea government (Ministry of Science, ICT \& Future Planning) (NRF2016R1A3B1908431). N.-G.P. acknowledges financial support from the National Research Foundation of Korea (NRF) grants funded by the Ministry of Science and ICT (MSIT) of Korea under contracts NRF-2014M3A6A7060583 (Global Frontier R\&D Programme on Center for Multiscale Energy System). The ${ }^{1} \mathrm{H}$ MAS NMR work was supported by the National Research Foundation of Korea (NRF) grant funded by the Ministry of Science and ICT to S.K. Lee (2017R1A2A1A17069511). A.S., S.N. and R.H.F. acknowledge funding and support from the SUNRISE project (EP/P032591/1), EPSRC and UKIERI project. S.N. would like to acknowledge funding and support from the Royal Society Newton International Fellowship.

\section{Author contributions}

H.K. and J.S.K. conceptualized the study. H.K., J.S.K., and J.-M.H. developed devices and performed measurements. Z.L., L.Z., and H.M.J. applied computational technique. H.K., J.S.K., and J.-M.H., M.P., I.-H.P., H.J.Y., M.-H.P., S.-H.J., Y.-H.K., J.-W.P., E.O., S.N., A.S., J.J.K., S.K.L., H.Y., K.P.L., N.-G.P. conducted the research and investigation process. H.K. and J.S.K., wrote the initial draft. H.K., J.S.K., J.-M.H., L.Z., S.K.L., H.Y., H.M.J., R.H.F., K.P.L., M.K.N., N.-G.P., and T.-W.L. reviewed and edited the paper. T.-W.L. supervised.

\section{Competing interests}

The authors declare no competing interests.

\section{Additional information}

Supplementary information is available for this paper at https://doi.org/10.1038/s41467020-17072-0.

Correspondence and requests for materials should be addressed to T.-W.L.

Peer review information Nature Communications thanks Jovana Milic and the other, anonymous, reviewer(s) for their contribution to the peer review of this work. Peer reviewer reports are available.

Reprints and permission information is available at http://www.nature.com/reprints

Publisher's note Springer Nature remains neutral with regard to jurisdictional claims in published maps and institutional affiliations.

\begin{abstract}
(c) (i)
Open Access This article is licensed under a Creative Commons Attribution 4.0 International License, which permits use, sharing, adaptation, distribution and reproduction in any medium or format, as long as you give appropriate credit to the original author(s) and the source, provide a link to the Creative Commons license, and indicate if changes were made. The images or other third party material in this article are included in the article's Creative Commons license, unless indicated otherwise in a credit line to the material. If material is not included in the article's Creative Commons license and your intended use is not permitted by statutory regulation or exceeds the permitted use, you will need to obtain permission directly from the copyright holder. To view a copy of this license, visit http://creativecommons.org/ licenses/by/4.0/.
\end{abstract}

(C) The Author(s) 2020 